Check for updates

Cite this: RSC Adv., 2018, 8, 39477

Received 3rd October 2018

Accepted 16th November 2018

DOI: $10.1039 / \mathrm{c} 8 \mathrm{ra} 08198 \mathrm{k}$

rsc.li/rsc-advances

\title{
Multiple 3D-QSAR modeling, e-pharmacophore, molecular docking, and in vitro study to explore novel AChE inhibitors $\uparrow$
}

\author{
Srabanti Jana, (D) Ankit Ganeshpurkar (iD) and Sushil Kumar Singh (D)*
}

\begin{abstract}
Ligand-based and energy-optimized structure-based approaches were considered to obtain excellent candidates as AChE inhibitors. The known AChE inhibitors were utilized to develop a pharmacophore hypothesis, HPRRR and X-ray crystallographic structures of AChE were used to produce three epharmacophore hypotheses viz. AHHRR, AHRR, and DHRR. Based on in silico approaches, we came across eight structurally diverse hits as non-competitive AChE inhibitors with good ADME properties. The best four hits, ZINC20592007, ZINC05354646, ZINC20649934, and ZINC39154782 were non-toxic, neuroprotective, and were selective AChE inhibitors $\left(\mathrm{IC}_{50}\right.$ values $482 \pm 1.88 \mathrm{nM}, 580 \pm 1.63 \mathrm{nM}, 854 \pm$ $2.65 \mathrm{nM}$, and $636 \pm 1.79 \mathrm{nM}$ respectively). The hits showed non-competitive inhibition of AChE at PAS site with attractive $K_{i}$ values $(0.21 \pm 0.027 \mu \mathrm{M}, 0.27 \pm 0.064 \mu \mathrm{M}, 0.3 \pm 0.018 \mu \mathrm{M}$, and $0.28 \pm 0.032 \mu \mathrm{M}$ for ZINC20592007, ZINC05354646, ZINC20649934, and ZINC39154782 respectively), and increased the cholinergic activity as well as inhibited $A \beta$ aggregation.
\end{abstract}

\section{Introduction}

Acetylcholinesterase (AChE), a $3.5 \mathrm{kDa}$ protein, is a member of the carboxylesterase family with an $\alpha / \beta$-hydrolase fold. ${ }^{1}$ The leading role of AChE is the hydrolysis of synaptic acetylcholine (ACh) and regulation of cholinergic neurotransmission in the body. It also plays a pivotal role in neuritogenesis, synaptogenesis, amyloidosis, dopamine neuron activation, regulation of apoptosis, nerve regeneration, hematopoiesis, and lymphocyte activation. ${ }^{2,3}$ The in vitro and in vivo exercises explain the relationship between amyloid precursor protein (APP) processing and cholinergic activation through muscarinic and nicotinic receptors. ${ }^{\mathbf{4 , 5}}$

Structurally, AChE consists of 'large central mixed $\beta$-sheets' surrounded by ' $15 \alpha$-helices'. ${ }^{6}$ The catalytic anionic site (CAS) is located at the bottom of a narrow gorge of AChE consisting of esteratic site (Ser203, Glu334, and His447) and anionic site (Trp86). Another site, named as peripheral anionic site (PAS) (consisting of Tyr72, Asp74, Tyr124, Trp286, and Tyr341 residues) is $20 \AA$ from the catalytic center. The aromatic residue's

Pharmaceutical Chemistry Research Laboratory, Department of Pharmaceutical Engineering \& Technology, Indian Institute of Technology (Banaras Hindu University), Varanasi-221005, India. E-mail: sksingh.phe@iitbhu.ac.in; Tel: +91-542-6702736/49

$\dagger$ Electronic supplementary information (ESI) available: Results of DFT study, graphs, and calculations of enzyme inhibition study, experimental procedure, and calculations of PAMPA study; Structures of known AChE inhibitors with test and training sets for development of pharmacophore; identified virtual hits alignment with their pharmacophores and interaction images of hits. See DOI: 10.1039/c8ra08198k ring creates $40 \%$ surface of the gorge and is located in the loop, thus presenting greater conformational flexibility. The Trp86 residue forms $\pi$-cation interaction with quaternary nitrogen of the ACh along with Phe $338 .^{7}$ The PAS of AChE acts as an adhesion site to non-amyloidogenic conformer of $\mathrm{A} \beta$ leading to its conformational change to produce amyloid fibrils. ${ }^{8}$ The Trp286 at the PAS site mimics response of the whole enzyme on amyloid formation. ${ }^{9}$ Further, AChE-A $\beta$ complexes induce neurotoxicity and trigger more neurodegeneration than $A \beta$ peptide alone. Thus, designing AChE inhibitor (AChEI) that blocks PAS of the enzyme will prevent $A \beta$ aggregation as well as enhance cholinergic transmission for treating Alzheimer's disease $(\mathrm{AD})$.

Both, $\beta$-amyloid protein $(\mathrm{A} \beta)$ and abnormally hyperphosphorylated tau (P-tau) can influence AChE overexpression in $\mathrm{AD} .{ }^{10}$ The improvement of cholinergic transmission by using AChEI may boost cognitive impairment of patients with schizophrenia, ${ }^{\mathbf{1 1}, 12}$ and Parkinson's disease (PD). ${ }^{13}$ The acetylcholine receptors at neuromuscular junction are reduced in myasthenia gravis (MG) ${ }^{\mathbf{1 4}}$ and AChEIs are considered essential for the treatment of MG. AChEIs can enhance cholinergic upregulation by weakening the effect of neuroinflammation via immunocompetent cells expressing $\alpha$-7-acetylcholine receptor (AChR). ${ }^{15}$

Butyrylcholinesterase (BuChE) level is increased by up to 2fold during mild to moderate $\mathrm{AD},{ }^{\mathbf{1 6}}$ and causes an imbalance between synthesis or synaptic release of ACh and its enzymatic hydrolysis. Therefore, AChEIs with BuChE inhibition property may provide better therapeutic value in neurological disorders. 
LIGAND BASED DRUG DESIGN

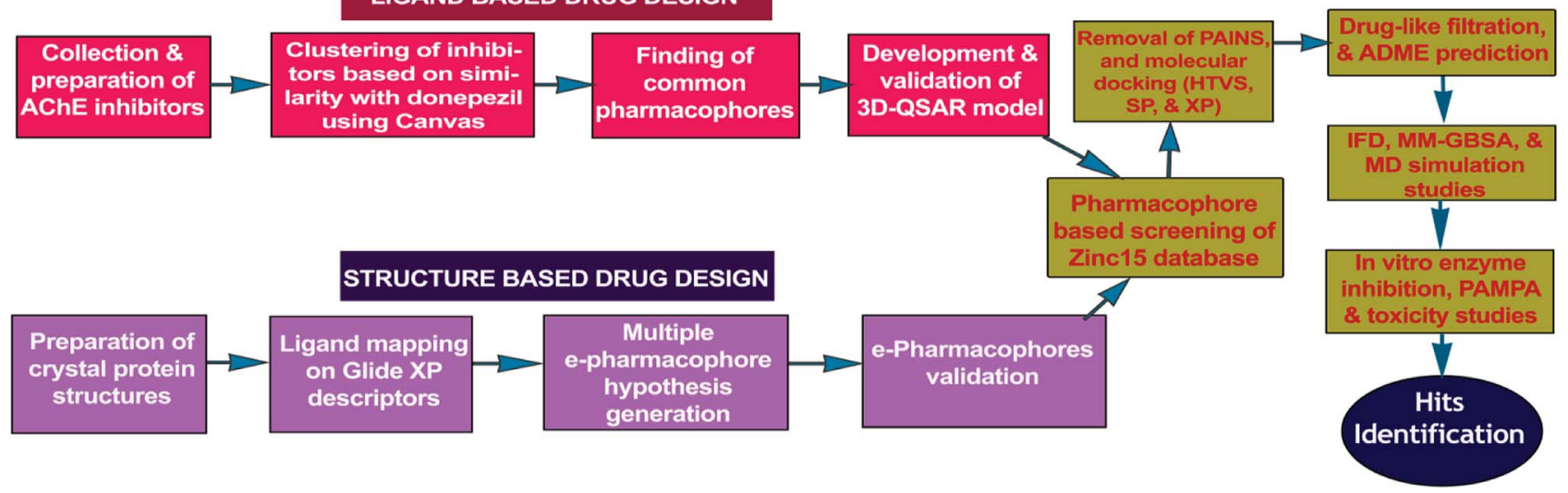

Fig. 1 Flowchart of hit identification based on ligand-based and structure-based pharmacophore models.

The drug discovery process is time-consuming and cumbersome, but the use of in silico approaches helps to identify better hits and scaffolds for a target. The pharmacophore modeling is a mathematical modeling technique which may help in the quick prediction of hits. The combination of ligand-based and structure-based pharmacophore models help in better productivity of outcome. ${ }^{17}$ Earlier researchers attempted to develop pharmacophore models and utilized them for virtual screening of database molecules to find new AChE inhibitors. $^{18-23}$

Present work combines both ligand-based (3D-QSAR) and energy optimized structure-based pharmacophore (epharmacophore) approaches for virtual screening of free 'ZINC15' database molecules. The hits, as AChE inhibitors, were recognized by utilizing HTVS and molecular docking studies of pharmacophore matched compounds after removal of panassay interference compounds (PAINS). ${ }^{24}$ The workflow of hit identification based on ligand-based 3D-QSAR and structurebased e-pharmacophore is explicit in Fig. 1.

The in vitro studies viz. enzyme inhibitions (AChE \& BuChE), enzyme kinetics (AChE), propidium iodide displacement from AChE, parallel artificial membrane permeability assay for blood-brain barrier (PAMPA-BBB), effects on cell viability and neuroprotectivity against apoptosis triggered by L-glutamate, approved and validated the outcome from the in silico study.

\section{Results and discussion}

\section{Development of ligand-based pharmacophore model}

Atom-based 3D-QSAR model was developed by using 142 datasets, which was divided into actives, inactives, and moderately actives. Total 163 hypotheses were generated and the best pharmacophore hypothesis, HPRRR, was selected on the basis of good survival activity (3.7), survival-inactive score (2.22), vector score (0.998), volume (0.862), selectivity (2.576), energy scores, best active alignment, and number of site matches (Table 1).

Hypothesis HPRRR: one hydrogen-bond acceptor, one positive ionizable group, and three aromatic rings showed the highest survival score. The developed 3D-QSAR pharmacophore model was statistically validated internally and externally to exhibit reliable predictions. We randomly selected 100 compounds in the training set and 42 in the test set to generate 3D-QSAR model. The statistical parameters were obtained by 'leave one out' (LOO) method and by partial least-square (PLS) analyze. HPRRR hypothesis showed better predictive ability, with PLS factor 5 than others (Table 2).

\section{Validation of ligand-based pharmacophore model}

At PLS factor 5, hypothesis HPRRR showed low SD value of 0.225 , RMSE of 0.409 , and $P$ value of $1.13 \times 10^{-64}$, and higher $R^{2}$ of 0.961 for the training set, and good $Q^{2}$ of 0.729 ; Person- $R$ of $0.857 ; F$ value of 465.7 for the test set. Therefore, HPRRR model had good predictivity at PLS factor 5 and was taken for further pharmacophore-based screening of database molecules. The pharmacophoric features of 3D-QSAR hypothesis are sketched in Fig. 2. The distance between pharmacophores was within the range of 2.174-11.329 $\AA$ (Table 3).

All the parameters for external validation of ligand-based pharmacophore model helped to select best model (Table 4). The correlation coefficient $\left(r^{2}\right)$ value of 0.922 , cross-validation

Table 1 The 3D-QSAR pharmacophore hypothesis with various scores

\begin{tabular}{lllllll}
\hline Hypothesis $^{a}$ & Survival score & Survival-inactive score & Site score & Vector score & Volume & Selectivity \\
\hline HPRRR & 3.7 & 2.22 & 0.84 & 0.998 & 0.862
\end{tabular}

${ }^{a} \mathrm{H}$, hydrogen bond donor; $\mathrm{P}$, positively ionizable group; and $\mathrm{R}$, aromatic ring. 
Table 2 PHASE 3D-QSAR and PLS statistics for internal validation of hypothesis

\begin{tabular}{|c|c|c|c|c|c|c|c|c|c|}
\hline Hypothesis & PLS factor & $\mathrm{SD}^{a}$ & $R^{2 b}$ & $F^{c}$ & $P$ & Stability & $\mathrm{RMSE}^{d}$ & $Q^{2 e}$ & Pearson- $\mathrm{R}^{f}$ \\
\hline HPRRR & 1 & 0.735 & 0.570 & 129.7 & $1.204 \times 10^{-19}$ & 0.933 & 0.651 & 0.314 & 0.588 \\
\hline & 2 & 0.482 & 0.817 & 216.5 & $1.706 \times 10^{-36}$ & 0.843 & 0.480 & 0.627 & 0.797 \\
\hline & 4 & 0.288 & 0.936 & 347.5 & $8.721 \times 10^{-56}$ & 0.702 & 0.437 & 0.691 & 0.834 \\
\hline & 5 & 0.225 & 0.961 & 465.7 & $1.13 \times 10^{-64}$ & 0.678 & 0.409 & 0.729 & 0.857 \\
\hline
\end{tabular}

${ }^{a}$ Standard deviation of the regression. ${ }^{b}$ The square of correlation coefficient. ${ }^{c}$ Variance ratio. ${ }^{d}$ Root-mean-square error. ${ }^{e}$ Squared $Q$ value for the predicted activities. ${ }^{f}$ Correlation between the predicted and observed activities for the test set.

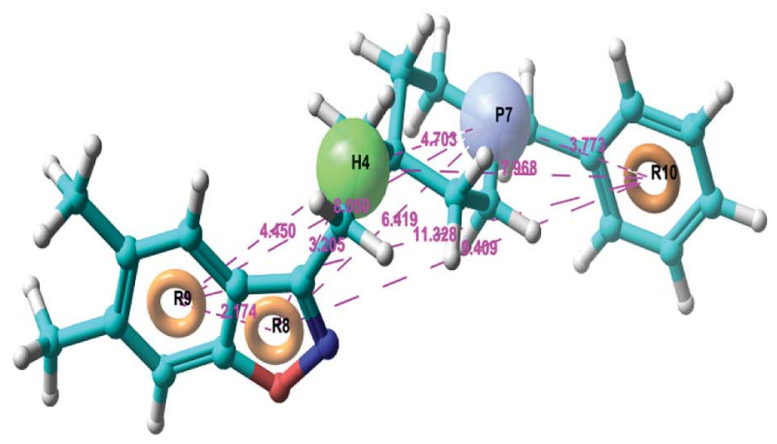

3D-QSAR-HPRRR

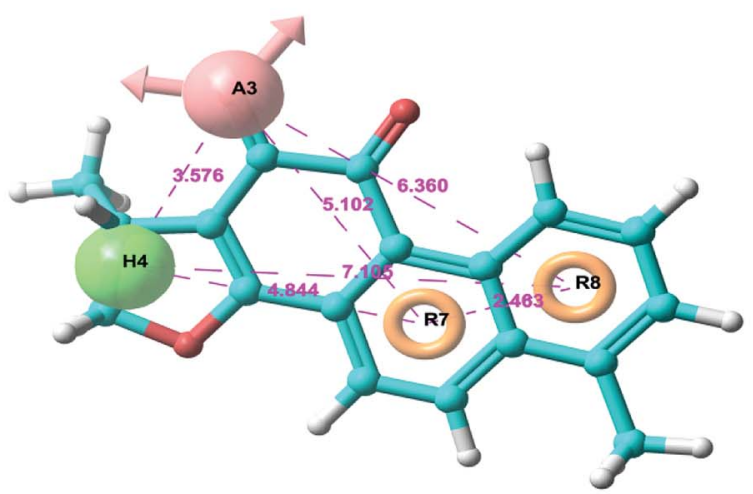

4MOE-AHRR

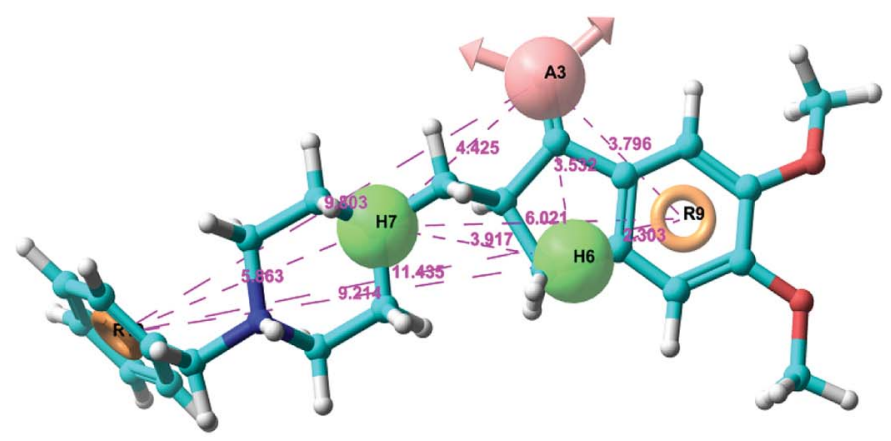

4EY7-AHHRR

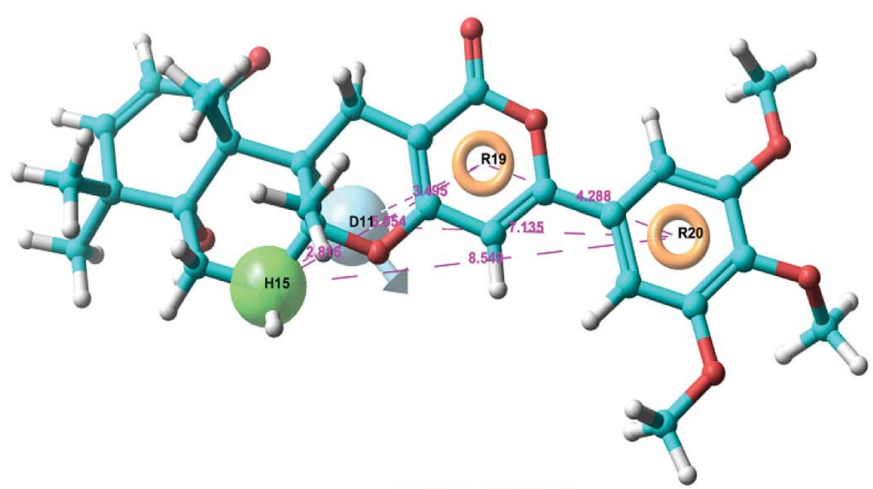

4MOF-DHRR

Fig. 2 3D-QSAR pharmacophore hypotheses and structure-based pharmacophores models with their respective crystal structures. (A) H-bond acceptor, pink sphere containing arrow; (D) $\mathrm{H}$-bond donor, sky blue sphere with arrow; $(H)$ hydrophobic group, green sphere; $(\mathrm{P})$ positive ionizable group, violet sphere; (R) aromatic ring, yellow circle.

coefficient $\left(r_{\mathrm{cv}}{ }^{2}\right)$ value of 0.919 , square of correlation coefficient value using the LOO method, $\left.\left(R_{\mathrm{m}(\mathrm{LOO})}\right)^{2}\right)$, of 0.834 , also helped to consider 3D-QSAR model as a better predictive model. The slopes of regression lines through origin ( $K$ and $K^{\prime}$ value) and substantial values of correlation coefficients $\left(R_{0}{ }^{2}\right.$ and ${R_{0}^{\prime}}^{2})$ were obtained from observed activity versus predictive activity plots (Fig. 3). The values were also within the limits and encouraged the model predictivity.

\section{Development of energy-optimized structure-based pharmacophore}

Total three human AChE (hAChE) crystal structures with a resolution between $2.0 \AA$ and $2.35 \AA$ and potent AChE inhibitory activity $\left(\mathrm{IC}_{50}\right.$ from 5.3 to $7 \mathrm{nM}$ and $K_{\mathrm{i}} 1.7$ to $700 \mathrm{nM}$ ) were selected for developing e-pharmacophore. Protein preparation wizard was used to prepare the proteins with an OPLS_2005 force field. After refinement, the protein structures with ligand interaction showed that donepezil (cocrystal of 4EY7) interacted with Trp86, and TRP286 by a pi-pi stacking, $\mathrm{H}$-bond interacted with Phe295, and pi-cationic with Phe338 residue. The dihydrotanshinone I (cocrystal of 4M0E) interacted with TRP286 by a pi-pi stacking, H-bond interaction was with Phe295; and territrem B (cocrystal of $4 \mathrm{M} 0 \mathrm{~F}$ ) interacted with TRP286 by a pi-pi stacking and $\mathrm{H}$-bond interaction was with Tyr124 at the PAS site (Fig. 4).

The refined cocrystal ligands were redocked onto the respective prepared protein structures to generate energyoptimized structure-based pharmacophore (e- 
Table 3 Distance between features of 3D-QSAR hypotheses and e-pharmacophores

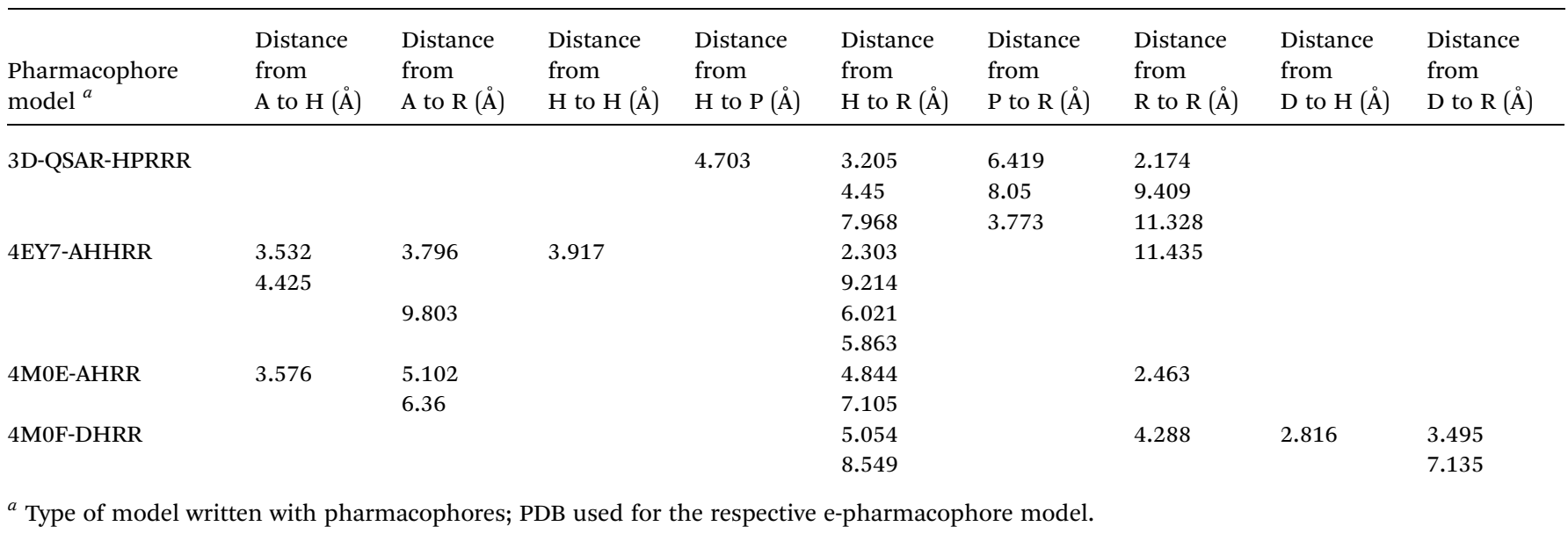

Table 4 External validation parameters for 3D-QSAR

\begin{tabular}{lll}
\hline $\begin{array}{l}\text { External validation } \\
\text { parameters }\end{array}$ & HPRRR & Limitations \\
\hline$r_{\mathrm{cv}}{ }^{2 a}$ & 0.919 & $r_{\mathrm{cv}}{ }^{2}>0.5$ \\
$r^{2 b}$ & 0.922 & $r^{2}$ close to 1 \\
$k$ value $^{c}$ & 0.990 & $0.85 \leq k \leq 1.15$ \\
$K^{\prime}$ value $^{d}$ & 1.008 & $0.85 \leq k \leq 1.15$ \\
$R_{0}^{2 e}$ & 0.916 & Close to $r^{2}$ \\
$R_{0}^{\prime 2 f}$ & 0.921 & Close to $r^{2}$ \\
$R_{\mathrm{m}(\text { LOO) }}{ }^{2 g}$ & 0.834 & $R_{\mathrm{m}(\text { LOO })}{ }^{2}>0.5$ \\
$r_{\text {pred }}{ }^{2 h}$ & 0.738 & $r_{\text {pred }}^{2}>0.5$
\end{tabular}

${ }^{a}$ Cross-validated coefficient. ${ }^{b}$ Correlation coefficient between actual and predicted values. ${ }^{c}$ Slope values of regression lines. ${ }^{d}$ Slope values of regression lines. ${ }^{e}$ Correlation coefficients for regression lines through origin. ${ }^{f}$ Correlation coefficients for regression lines through origin. ${ }^{g}$ Modified squared correlation coefficient using LOO method.

${ }^{h}$ Predictive correlation coefficient value.

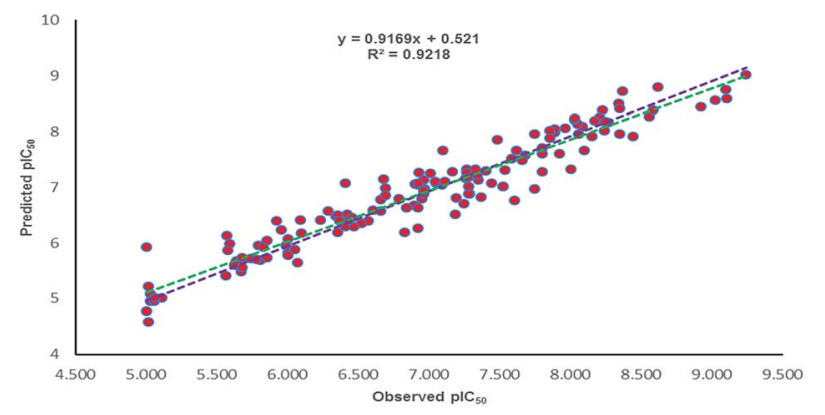

Fig. 3 Plot of predicted $\mathrm{plC}_{50}$ versus observed $\mathrm{pIC}_{50}$ of $\mathrm{AChE}$ inhibitors developed by model HPRRR with regression lines (original regression lines represented in green break line and regression lines with intercept zero in purple break line).

pharmacophore). The root-mean-square deviation (rmsd) was less than $1 \AA$ for all the three cocrystal ligands. The epharmacophore hypotheses were generated by mapping Glide XP energetic terms onto pharmacophore sites, which were calculated from the structural and energy information between protein and ligand. Initially, the numbers of pharmacophore sites were set up to 10 for each of crystal structures for pharmacophore generation, but numbers of pharmacophore sites were selected, for the best hypothesis, on the basis of validation parameters. The total number of pharmacophore sites for each cocrystal ligand before energy-based site selection and selected sites for hypothesis generation for the three crystal structures with pharmacophoric feature scores are given in Table 5 . The epharmacophore models generated were AHHRR with 5 sites from 4EY7, AHRR with 4 sites from 4M0E, and DHRR with 4 sites from $4 \mathrm{M} 0 \mathrm{~F}$ crystal structure (Fig. 2). In these pharmacophore modeling, A stranded for $\mathrm{H}$-bond acceptor, $\mathrm{D}$ for $\mathrm{H}$-bond donor, $\mathrm{H}$ for hydrophobic group, $\mathrm{R}$ for aromatic ring. The distance between e-pharmacophore features was within range of 2.303-11.435 А̊ (Table 3).

\section{Validation of energy-optimized structure-based pharmacophore}

The database, consisting of 1053 compounds using 1000 druglike decoys and 53 known AChE inhibitors, was utilized for epharmacophore validation., We evaluated enrichment factor (EF) and Goodness of hit score (GH) utilizing Güner-Henry scoring method To validate the e-pharmacophores (Table 6). The values of GH over 0.5 and EF higher than 10, ensured the suitability of pharmacophores for further pharmacophorebased virtual screening.

\section{Pharmacophore matched screening and removal of pan-assay interference compounds (PAINS)}

Pharmacophore matched molecules were separated out from the total 3530990 ZINC15 database compounds (without known AChE inhibitors) by utilizing advance pharmacophore screening option of PHASE. The fitness value is a measure of how well the ligand fits with the pharmacophore. The hits with high fitness value of more than 1.5 are probably very active inhibitors. We employed the validated three e-pharmacophores, and one ligand-based pharmacophore to screen the database of 2000 AChE inhibitor molecules by each model. 


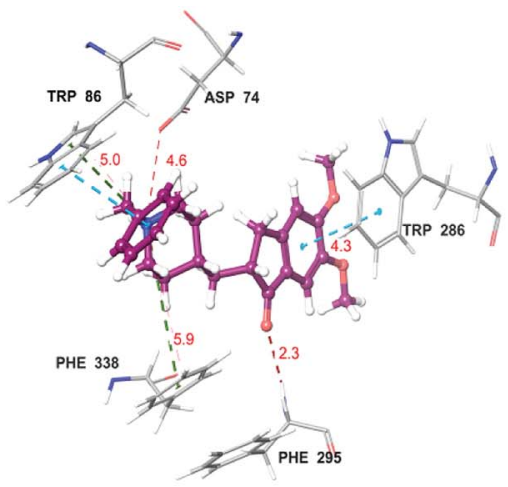

4EY7

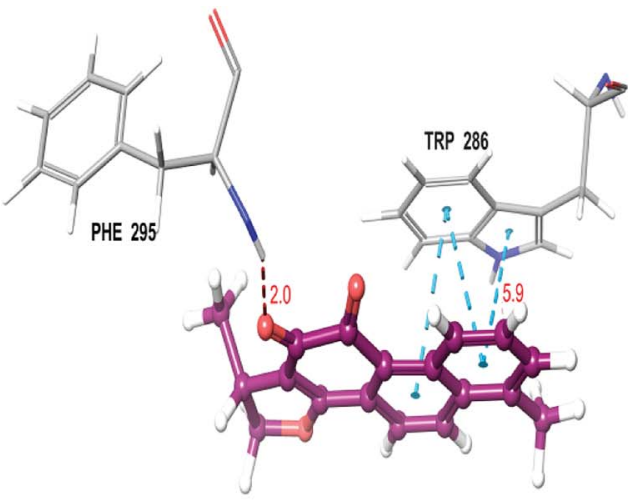

4MOE

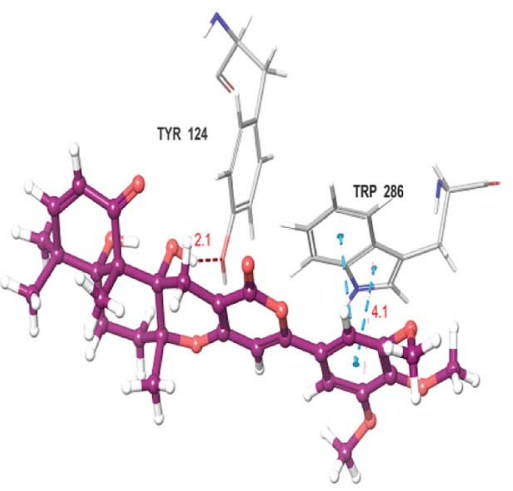

4MOF

Fig. 4 Crystal structures of AChE with cocrystal ligands (purple color) and bonding interactions.

Table 5 e-Pharmacophore hypotheses with features scores

\begin{tabular}{lllll}
\hline PDB & No. of possible site & No. of accepted site & Hypotheses $^{a}$ & Pharmacophore features with score \\
\hline 4EY7 & 6 & 5 & AHHRR & H7: -1.64, H6: -1.5, R10: -1.48, R9: -1.2, A3: -0.73 \\
4M0E & 4 & 4 & AHRR & A3: -1.7, R7: 1.62, H4: -1.5, R8: -1.13 \\
4M0F & 7 & 4 & DHRR & R19: -1.5, D11: -1.49, R20: -1.3, H15: -0.66
\end{tabular}

${ }^{a}$ A, H-bond acceptor; D, hydrogen bond donor; H, hydrophobic group; P, positively ionizable group; R, aromatic ring.

Table 6 Validation of e-pharmacophores with the Güner-Henry scoring method using a dataset consisting of total 1053 compounds with 53 total actives compounds

\begin{tabular}{llll}
\hline Parameters & $4 \mathrm{EY7}$ & $4 \mathrm{M} 0 \mathrm{E}$ & $4 \mathrm{M} 0 \mathrm{~F}$ \\
\hline $\mathrm{Ht}^{a}$ & 74 & 69 & 71 \\
$\mathrm{Ha}^{b}$ & 41 & 37 & 43 \\
$\mathrm{EF}^{c}$ & 11.008 & 10.654 & 12.033 \\
$\mathrm{GH}^{d}$ & 0.589 & 0.558 & 0.639
\end{tabular}

${ }^{a}$ Total hits. ${ }^{b}$ Active hits. ${ }^{c}$ Overall enrichment factor. ${ }^{d}$ Goodness of hit score.

The reactivity towards proteins to develop poor potentiality or known toxicity of molecules, i.e., PAINS was removed from pharmacophore matched compounds by using RDKit, ZINC, and FAF-Drugs 4 server. Only less than $1 \%$ of PAINS compounds were removed and mild PAINS were ignored for virtual screening.

\section{High throughput virtual screening (HTVS) and molecular docking}

High throughput screening of PAINS removed pharmacophore matched database and was a fruitful resource for initial hit identification. The number of hits from pharmacophore-based virtual screening and process of selection with their respective PDB are presented in Table 7.

Molecular XP docking was performed for all the outcome HTVS retrieves with $4 \mathrm{MOE}$ crystal structure to compare docking scores of hits with reference donepezil. We found that 55 molecules were having docking score more than -9.0. Finally, eight compounds with structural diversity, PAINS free (except ZINC20592007, a PAINS-ok molecule), better docking scores $(-12.87$ to -10.74$)$ and Glide energies $(-56.48$ to $-42.16 \mathrm{kcal} \mathrm{mol}^{-1}$ ) than donepezil were selected for further studies (Table 8). The hits outcome with respect to pharmacophore models are listed in ESI (Table S1 $\dagger$ ). The protein-ligand interactions with types of interactions and interacting residues with hits and donepezil are included in Table 8. The chemical structures of hits are sketched in Fig. 5 and ligandprotein interactions are pictured in Fig. 6; hits are represented in yellowish green, interacting amino acid residues of protein in gray, $\mathrm{H}$-bond in red, pi-cationic interaction in green and pi-pi stacking in cyan color. The presence of more hydroxyl, keto, secondary amine and nitrogen-containing hetero aromatics in hits were responsible for formation of hydrogen bond and more docking score than donepezil.

The hits mainly bind at PAS site of AChE through H-bond interactions with Phe295, Tyr337, and Phe338 residues (within 1.8-2.3 Å bond distance), pi-pi stacking with Trp286, His 287, Phe297, and Tyr341 residues (within 3.8-5.1 Å distance), and picationic or salt bridge interactions with Asp74, Tyr341, and Trp286 residues (within 1.9-5.6 ̊ distance). Keto group of all hits, except ZINC77161317 and ZINC39154782 were formed Hbonding with Phe295 and Ser293, were formed hydrogen bonding with Phe295 reside of protein. Aromatic group of hits were produced $\pi-\pi$ stacking interaction with Trp286, except ZINC39154782 interacting with Phe297. 
Table 7 Number of compounds retrieved at each stage of screening of dataset

\begin{tabular}{|c|c|c|c|c|c|}
\hline $\begin{array}{l}\text { Pharmacophore } \\
\text { models }\end{array}$ & PDB & HTVS hits & SP hits & XP hits & No. of selected hits \\
\hline AHHRR & $4 \mathrm{EY7}$ & 1991 & 200 & 20 & 1 \\
\hline DHRR & $4 \mathrm{M} 0 \mathrm{~F}$ & 1993 & 200 & 20 & 2 \\
\hline HPRRR & $4 \mathrm{M} 0 \mathrm{~F}$ & 1945 & 200 & 20 & 3 \\
\hline
\end{tabular}

Table 8 Hit molecules with their Glide docking score, number of $\mathrm{H}$-bonds, interaction with essential amino acids, IFD docking score, and AutoDock binding energy

\begin{tabular}{|c|c|c|c|c|c|c|c|}
\hline Title & $\begin{array}{l}\text { Glide } \\
\text { docking } \\
\text { score }\end{array}$ & $\begin{array}{l}\mathrm{H}- \\
\text { bond }\end{array}$ & $\begin{array}{l}\text { Glide } \\
\text { energy }\end{array}$ & Residue interactions & $\begin{array}{l}\text { MM- } \\
\text { GBSA- } \\
\Delta G_{\text {Bind }} \\
\end{array}$ & $\begin{array}{l}\text { IFD } \\
\text { docking } \\
\text { score }\end{array}$ & $\begin{array}{l}\text { AutoDock } \\
\text { binding } \\
\text { energy }\end{array}$ \\
\hline ZINC72451013 & -12.87 & 3 & -51.63 & $\begin{array}{l}\text { Asp } 74^{a}, \text { Phe295 }, \text { Arg296 }, \text { Tyr337 } \\
\text { Tyr341 }\end{array}$ & -65.17 & -11.53 & -10.34 \\
\hline ZINC20649934 & -12.65 & 2 & -50.50 & $\begin{array}{l}\text { Asp } 74^{a}, \text { Trp } 286^{c}, \text { Phe } 295^{b}, \text { Tyr } 337^{b}, \\
\text { Tyr341 }\end{array}$ & -94.16 & -10.77 & -11.69 \\
\hline ZINC05354646 & -11.93 & 1 & -42.16 & Trp286 $6^{c, d}$, Phe $295^{b}$ & -73.30 & -12.64 & -9.85 \\
\hline ZINC79331983 & -11.40 & 4 & -54.98 & Asp $74^{a, b}$, Phe295 $5^{b}$, Tyr $337^{b}$ & -79.39 & -11.09 & -10.65 \\
\hline ZINC20592007 & -11.26 & 2 & -47.79 & Tyr $^{2} 2^{b}$, Trp286 $6^{c, d}$, His $287^{c}$, Phe $295^{b}$ & -79.10 & -12.78 & -10.77 \\
\hline ZINC77161317 & -11.02 & 1 & -51.02 & Trp286 $6^{d}$ Phe295 $5^{b}$ Phe $297^{c}$ & -69.09 & -12.699 & -9.75 \\
\hline ZINC58160603 & -10.93 & 1 & -49.16 & $\operatorname{Trp} 286^{d}$, Phe295 $5^{b}$ & -80.61 & -11.85 & -9.32 \\
\hline ZINC39154782 & -10.74 & 3 & -49.28 & $\begin{array}{l}\text { Asp } 74^{a, b}, \text { Try } 124^{c}, \text { Ser } 293^{b}, \text { Tyr337 } \\
\text { Tyr } 341^{d} \text {, Phe } 297^{c}, \text { Phe } 338^{c}\end{array}$ & -76.11 & -11.88 & -10.54 \\
\hline Donepezil & -8.24 & 1 & -43.89 & Tyr $124^{d}$, Trp $286^{c}$, Phe $295^{b}$, Phe $338^{d}$ & -62.30 & -8.91 & -9.64 \\
\hline
\end{tabular}

${ }^{a}$ Salt bridge. ${ }^{b}$ H-bond interaction. ${ }^{c}$ Pi-Pi stacking. ${ }^{d}$ Pi-cation interaction.<smiles>CCN1CCC[C@H]1CNC(=O)c1ccc(-c2ccc(C)cc2)[nH]c1=O</smiles>

ZINC05354646<smiles>O=c1oc2c(CNCCc3cccc(F)c3)c(O)ccc2c2c1CCC2</smiles>

ZINC20592007<smiles>O=c1cc(CN2CCCC[C@H]2c2nc3ccccc3s2)nc2sccn12</smiles>

ZINC58160603

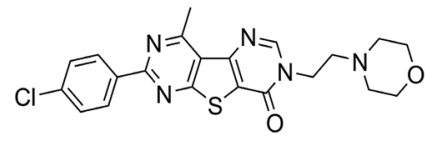

ZINC20649934<smiles>CN=C(NCCn1c(C)nc2ccccc21)NCc1ccc2c(c1)OCO2</smiles>

ZINC79331983<smiles>CC(C)c1nccn1CCN1CCN(C(=O)CN2C(=O)c3ccccc3C2=O)CC1</smiles>

ZINC77161317<smiles>COc1ccc(C2N=NC(NCCc3c[nH]c4ccc(C)cc34)NC2=O)cc1</smiles>

ZINC39154782
Fig. 5 Structures of final hits with zinc database ids.

\section{Induced fit docking}

The IFD scores of hits were close to the Glide XP docking scores (Table 8). The conformations generated from the IFD were little different from the docked poses produced from the rigid receptor docking. The Glide-based model gave an RMSD of $5.2 \AA$ when compared to the native pose in the crystal structure. The IFD docking pose and score were supported by the binding positions, affinity, and stability of hits.

\section{Calculation of prime MM-GBSA}

To predict the binding mode and binding free energy $\left(\Delta G_{\text {bind }}\right)$, the Prime MM-GBSA simulation was calculated for AChE-hits and AChE-cocrystal ligand complexes utilizing Maestro 10.1 (Table 8). All the hits showed better $\Delta G_{\text {bind }}$, and ZINC20649934 provided highest $\Delta G_{\text {bind }},-94.16 \mathrm{kcal} \mathrm{mol}^{-1}$. The binding free energy determination, based on Prime MM-GBSA, established the stability of AChE-hits complexes.

\section{Docking with AutoDock}

The AutoDock binding energy of the hits was calculated and presented in Table 8 . The binding energy of final eight hits was between -9.32 to $-11.69 \mathrm{kcal} \mathrm{mol}^{-1}$. The prediction of results was fully supported Glide XP docking and IFD results (Table 8). All the hits displayed similar binding affinity and docking pose with 4M0E utilizing AutoDock and Glide (ESI, Fig. S1 and S2 $\dagger$ ). 

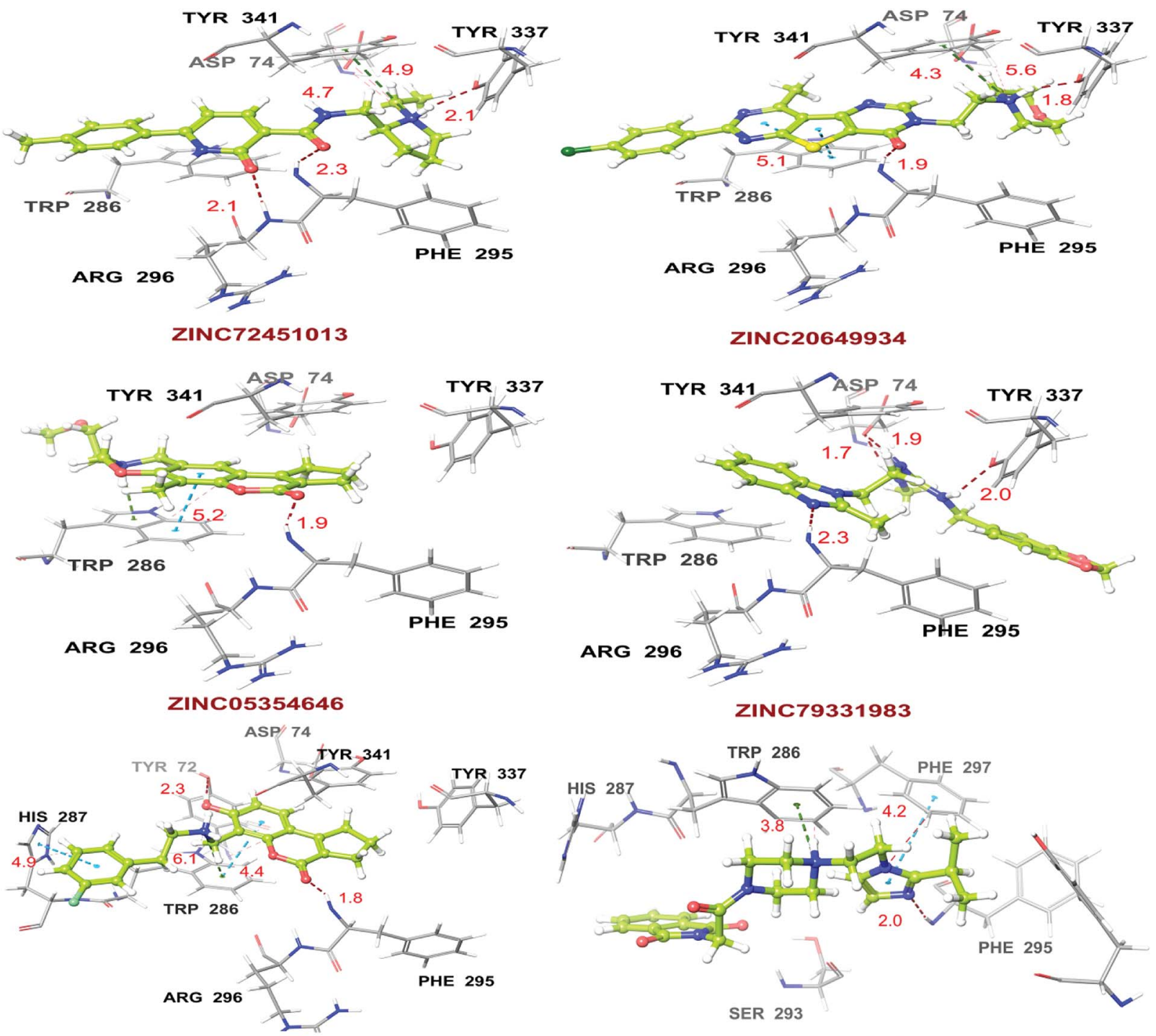

ZINC20592007

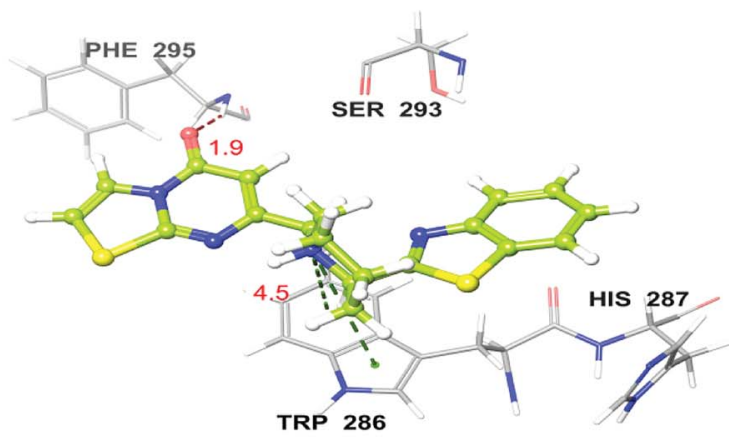

ZINC58160603

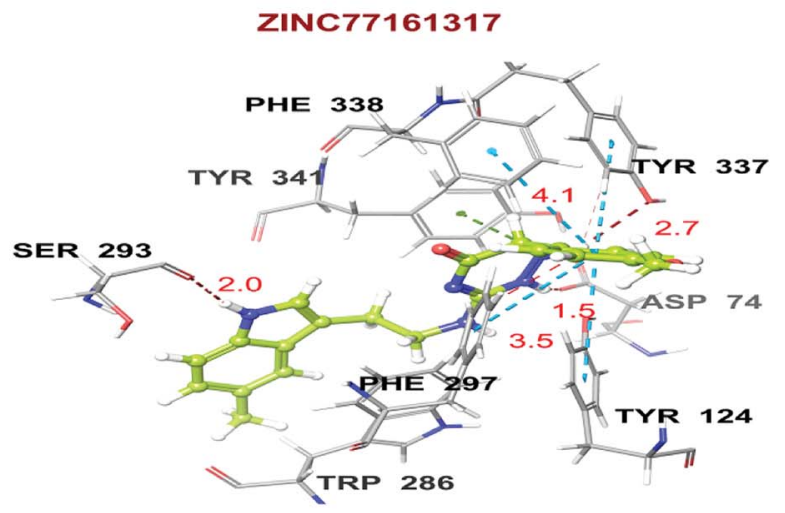

ZINC39154782

Fig. 6 Docking poses of ZINC72451013, ZINC20649934, ZINC05354646, ZINC79331983, ZINC20592007, ZINC77161317, ZINC58160603, and ZINC39154782 with AChE crystal structure; hits represented in yellowish green, residues in gray, $\mathrm{H}$-bond in red, pi-cationic interaction in green and pi-pi stacking in cyan color.

\section{Predicted ADME properties}

The predictions of drug-likeness and pharmacokinetics including absorption, distribution, metabolism, and excretion (ADME) were performed by utilizing QikProp tools of Maestro,
Schrödinger. We evaluated physiochemically descriptors and pharmaceutically relevant properties of hits to analyze druggable properties (Table 9). All the hit molecules showed a good partition coefficient (QP log Po/w) values (1.6 to 3.854), which 
Table 9 Hit molecules with their physiochemical descriptors determined by Qikprop tools

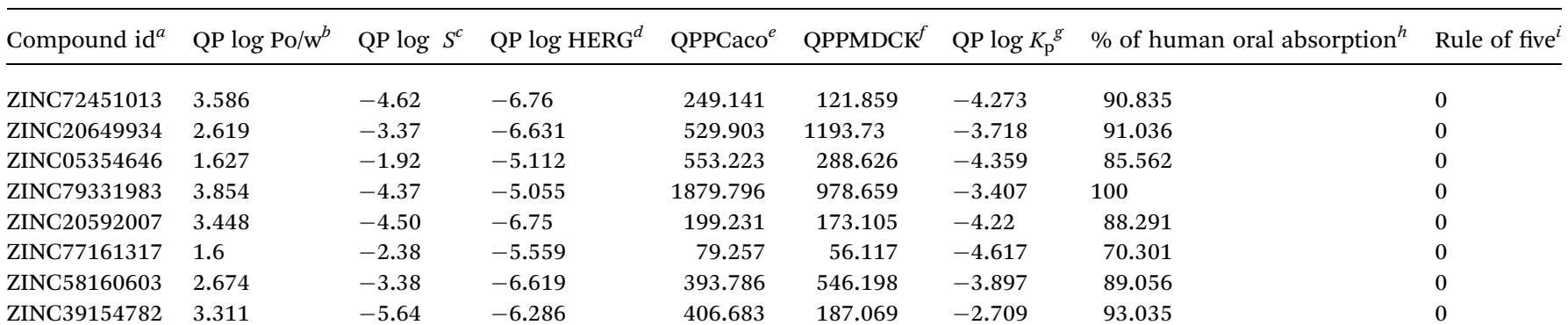

ZINC39154782 3.311

${ }^{a}$ Zinc database compound id. ${ }^{b}$ QP $\log$ Po/w for octanol/water $(-2.0$ to 6.5$) .{ }^{c}$ QP $\log S$ : predicted aqueous solubility, $S$ in mol dm ${ }^{-3}$ (-6.5 to 0.5 ). ${ }^{d} \log$ HERG: HERG K+ channel blockage $(<-5) .{ }^{e}$ Apparent Caco-2 cell permeability $\left(\mathrm{nm} \mathrm{s}^{-1}\right)(<25$ poor, $>500$ great $) .{ }^{f}$ Apparent MDCK permeability $\left(\mathrm{nm} \mathrm{s}^{-1}\right)\left(<25\right.$ poor, $>500$ great). ${ }^{g} \mathrm{QP} \log K_{\mathrm{p}}$ : skin permeability. ${ }^{h} \%$ human oral absorption $\left(>80 \%\right.$ is high and $<25 \%$ is poor). ${ }^{i}$ Rule of five: no. of violations of Lipinski's rule of five ( 0 is good and 4 is bad).

Table 10 Inhibitory activity on AChE (electric eel) and BChE (horse serum) and propidium competition assay results

\begin{tabular}{|c|c|c|c|c|c|c|}
\hline Compounds & $\mathrm{IC}_{50} \mathrm{AChE}^{a}(\mathrm{nM})$ & $\mathrm{IC}_{50} \mathrm{BChE}^{a}(\mathrm{nM})$ & Selectivity $^{b}(\mathrm{AChE} / \mathrm{BChE})$ & \multicolumn{3}{|c|}{ Propidium displacement (\%) } \\
\hline ZINC05354646 & $580 \pm 1.63$ & $147424 \pm 6.66$ & 254.2 & 0 & 42 & 57 \\
\hline ZINC20649934 & $854 \pm 2.65$ & $148654 \pm 6.24$ & 174.1 & 0 & 25 & 58 \\
\hline ZINC39154782 & $636 \pm 1.79$ & $128064 \pm 5.13$ & 201.4 & 29 & 58 & 100 \\
\hline
\end{tabular}

were critical for absorption and distribution of drugs. Factor QPPCaco, indicating permeability of these hits, was in the range of 79.257 to 1879.796 , where QPPCaco was a predicted apparent Caco-2 cell permeability in $\mathrm{nm} \mathrm{s}^{-1}$ value, a key factor for the estimation of cell permeability in biological membranes.

All the hits successfully passed the entire pharmacokinetic requirements for a drug-like compound and were within the acceptable range as defined for human use. Overall, the percentage of human oral absorption for the compounds were between 70.301 to $100 \%$, their water solubility (QP $\log S$ ) ranged between -1.919 to -6.373 , pMDCK (cell permeable parameter) values were between 56.117 to 1193.73 , skin permeability $\left(\log K_{\mathrm{p}}\right)$ values were within -2.709 to $-4.898 ; p \log$ HERG (K+ channel blockage) values were less than -5 . Additional parameters i.e., molecular weight, $\mathrm{H}$-bond donors, $\mathrm{H}$-bond acceptors, and $\log P$ according to Lipinski's rule of five, were also evaluated for their drug-like behavior. Thus, hits with better binding interaction and good predicted pharmacokinetic properties were considered for in vitro studies.

\section{Density functional theory}

The HOMO and LUMO of chemical compounds are crucial indicators of their reactivity and also stability of ligand-receptor interactions. ${ }^{25}$ The stability of interactions is inversely correlated to energy gap between HOMO and LUMO orbitals. The orbital energy of all energetically stable hit molecules was calculated by using a DFT method. The high value of HOMO energy is likely to indicate the tendency of molecule to donate electrons to an appropriate acceptor molecule with LUMO. The correlation of HOMO energies with $\mathrm{IC}_{50}$ data suggested that the HOMO of inhibitor might transfer its electrons to less energy, LUMO, of some amino residues in the active site of the enzyme. The calculated DFT properties of all hits are given in ESI (Table S2 $\dagger$ ). The HOMO-LUMO energy gaps of hits were minimal, and between -0.182 to $-0.012 \mathrm{eV}$. Leaser HOMO-LUMO energy gap facilitated electron(s) density exchanging properties or encouraged some interaction(s). The mean ESP indicated electron density distribution around nuclei of the molecules and was

Table 11 Permeability, Pe $\left(10^{-6} \mathrm{cms}^{-1}\right)$ determined by BBB-PAMPA study of hit compounds

\begin{tabular}{lccc}
\hline Compounds & $\operatorname{Pe}_{(\exp )}{ }^{a}\left[10^{-6} \mathrm{~cm} \mathrm{~s}^{-1}\right]$ & $\operatorname{Pe}_{(\text {actual })}^{a}\left[10^{-6} \mathrm{~cm} \mathrm{~s}^{-1}\right]$ & \\
\hline ZINC20592007 & $5.00 \pm 0.3$ & $5.7 \pm 0.20$ & CNS+ \\
ZINC05354646 & $7.8 \pm 0.25$ & $9.39 \pm 0.36$ & CNS+ \\
ZINC20649934 & $3.74 \pm 0.136$ & $5.45 \pm 0.79$ & CNS+ \\
ZINC39154782 & $4.69 \pm 0.2$ & $5.29 \pm 0.31$ & CNS+
\end{tabular}

${ }^{a}$ Data expressed as mean \pm SEM of three independent experiments. ${ }^{b}$ CNS + indicates good passive CNS permeation. 


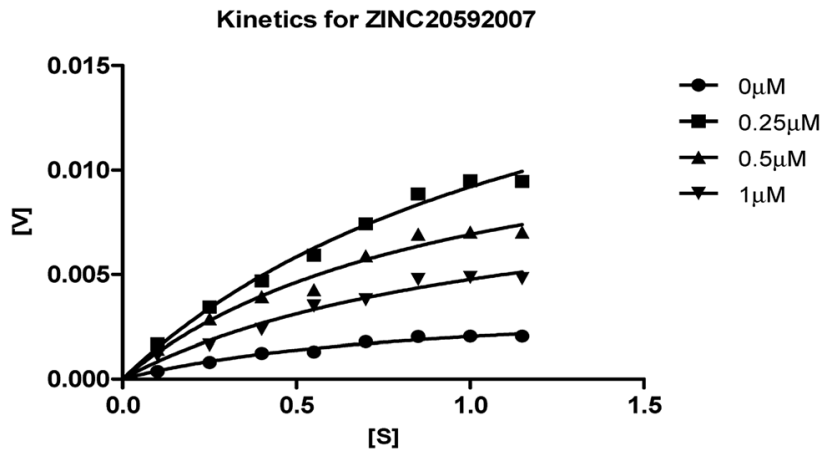

Fig. 7 Michaelis-Menten kinetics curve resulting from velocity of AChE activity with different substrate concentrations $(0.15-1.15 \mu \mathrm{M})$ in absence and presence of $0.25,0.5$ and $1 \mu \mathrm{M}$ of ZINC20592007.

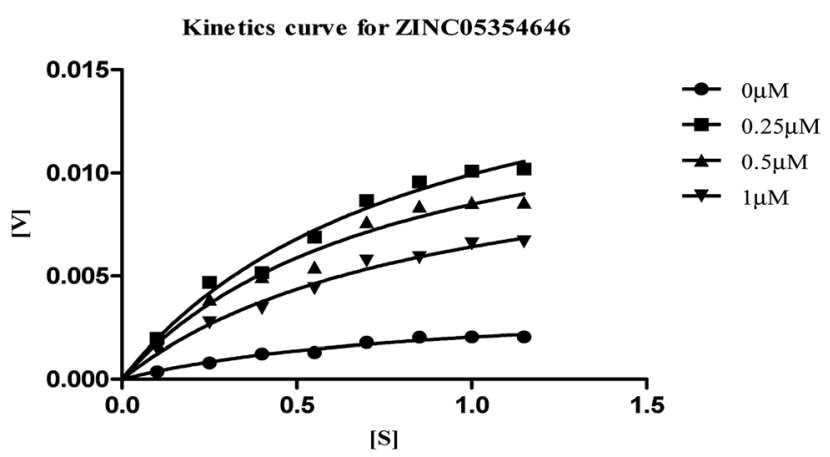

Fig. 8 Michaelis-Menten kinetics curve resulting from velocity of AChE activity with different substrate concentrations $(0.15-1.15 \mu \mathrm{M})$ in absence and presence of $0.25,0.5$ and $1 \mu \mathrm{M}$ of ZINC05354646.

between -0.22 to $1.89 \mathrm{kcal} \mathrm{mol}^{-1}$. The ESP data indicated that most of the hits contained both low and high electron density sites in a molecule. The upper and low electron density regions may correspond to the hydrogen bonding between the hits and enzyme.

\section{In silico AChE selectivity study}

We performed Glide XP docking study against BuChE by utilizing 4BDS crystal structure to estimate selectivity of hits

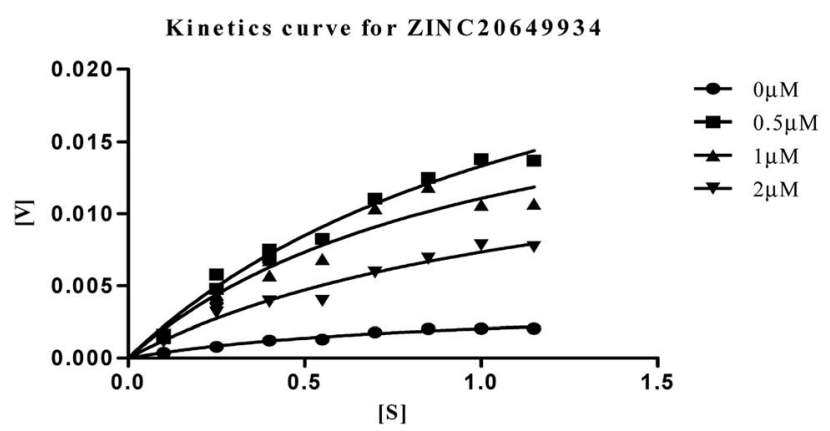

Fig. 9 Michaelis-Menten kinetics curve resulting from velocity of AChE activity with different substrate concentrations $(0.15-1.15 \mu \mathrm{M})$ in absence and presence of 0.5, 1 and $2 \mu \mathrm{M}$ of ZINC20649934.

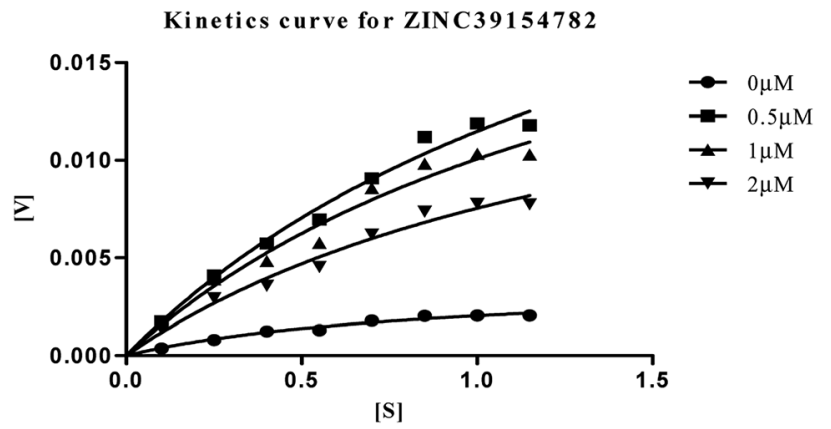

Fig. 10 Michaelis-Menten kinetics curve resulting from velocity of AChE activity with different substrate concentrations $(0.15-1.15 \mu \mathrm{M})$ in absence and presence of $0.5,1$ and $2 \mu \mathrm{M}$ of ZINC39154782.

towards AChE. All the hits had BuChE binding affinity with more selectivity towards AChE (ESI Table S3†). The hit ZINC05354646 showed lowest Glide docking score $(-5.66)$, and next lowest score compound was ZINC20649934 (-5.85) against BuChE crystal structure, 4BDS. The BuChE binding property of hits with AChE inhibition improved the therapeutic property of hits for cholinergic activity. The hits had moderate BuChE binding affinity which may improve cholinergic activity.

\section{In vitro inhibition of $\mathrm{AChE}$ and $\mathrm{BuChE}$}

Four hits (ZINC20592007, ZINC05354646, ZINC20649934, and ZINC39154782) were selected, based on Glide docking score, AutoDock energy, AChE selectivity, PAS site selectivity, ADME properties, and interesting structural features for further in vitro studies. ZINC20592007 contains a 2,3-dihydrocyclopenta[c] chromen-4(1H)-one fused nucleus, which is PAINS-ok (mannich-A type) molecule. ZINC05354646, a 2,3,9,10-tetrahydro-8H-cyclopenta[3,4]chromeno[6,7-e][1,3] oxazin-4(1H)-one fused compound, is PAINS free with similar scaffold of ZINC20592007. ZINC20649934 has thieno[2,3-d:4,5-d']dipyrimidin-4(3H)-one nucleus with attached morpholine ring to ethylene linker. ZINC39154782 contains 1,2,4-triazin-5(6H)-one with indole ring attached through an ethyl amino linker (Fig. 5).

The selected hits were evaluated for their anti-cholinesterase (anti-ChE) activity. AChE and BuChE inhibition activities were evaluated by the method described by Ellman, ${ }^{26}$ wherein donepezil was used as reference standard. Compound ZINC20592007 exhibited higher AChE inhibitory activity than ZINC05354646, ZINC20649934, and ZINC39154782 [IC I0 $_{\text {values }}$ $(\mathrm{nM})$ of $482 \pm 1.88,580 \pm 1.63,854 \pm 2.65$, and $636 \pm 1.79$, respectively (Table 10)]. All the hits has selective AChE inhibitory activity than BuChE enzyme (Table 10). The half maximal enzyme inhibitory concentration $\left(\mathrm{IC}_{50}\right)$, a measure of potency of hits inhibiting AChE and BuChE, was calculated by constructing a dose-response curve (ESI, Fig. S3†) by utilizing GraphPad Prism 5.0.

The mechanism of AChE enzyme inhibition of the four hits was determined by an enzyme kinetic study. Lineweaver-Burk reciprocal plots were generated by plotting reciprocal of reaction rates and reciprocal of substrate concentrations using 
Table 12 Cell viability, and neuroprotection of hit molecules in human neuroblastoma SH-SY5Y cell line

\begin{tabular}{|c|c|c|c|}
\hline \multirow[b]{2}{*}{ Compounds } & \multicolumn{2}{|c|}{ Cell viability ${ }^{a}(\%)$} & \multirow{2}{*}{$\frac{\text { Neuroprotection }^{b}(\%)}{25 \mu \mathrm{M}}$} \\
\hline & $50 \mu \mathrm{M}$ & $100 \mu \mathrm{M}$ & \\
\hline ZINC20592007 & $98.0 \pm 0.34$ & $95.3 \pm 0.32$ & $18.2 \pm 0.086$ \\
\hline ZINC05354646 & $90.2 \pm 0.39$ & $88.7 \pm 0.77$ & $20.0 \pm 0.061$ \\
\hline ZINC20649934 & $94.8 \pm 0.49$ & $93.0 \pm 0.45$ & $67.8 \pm 0.013$ \\
\hline ZINC39154782 & $98.6 \pm 0.55$ & $97.1 \pm 0.08$ & $26.3 \pm 0.077$ \\
\hline
\end{tabular}

${ }^{a}$ Percentage cell viability of SH-SY5Y cells exposed at relatively high concentrations $(50 \mu \mathrm{M}$ and $100 \mu \mathrm{M})$ of test compounds. ${ }^{b}$ Percentage neuroprotection of SH-SY5Y cells at relatively lower concentrations (25 $\mu \mathrm{M})$ of test compounds against L-glutamate $(100 \mu \mathrm{M})$. different concentrations of hit molecules. Michaelis-Menten kinetics curve resulting from velocity of AChE activity with varying concentrations of substrate $(0.15-1.15 \mu \mathrm{M})$ in absence and presence hit molecules $(0.25,0.5$ and $1 \mu \mathrm{M}$ of ZINC20592007 and ZINC05354646, and 0.5, 1, and $2 \mu \mathrm{M}$ for ZINC20649934, and ZINC39154782) are shown in Fig. 7, 8, 9, and 10 respectively. The $K_{\mathrm{i}}$ values of hits were determined by Yonetani-Theorell method from Lineweaver-Burk plots and presented in ESI as Fig. S4-S7† for ZINC20592007, ZINC05354646, ZINC20649934, and ZINC39154782 respectively.

The plots revealed that with increasing the concentrations of inhibitor, an increase in slope (decreased $V_{\max }$ ) and the intercept (higher $K_{\mathrm{m}}$ ) occurred. The lower apparent value of $V_{\max }$ in Michaelis-Menten plot to increase, decrease, or leave
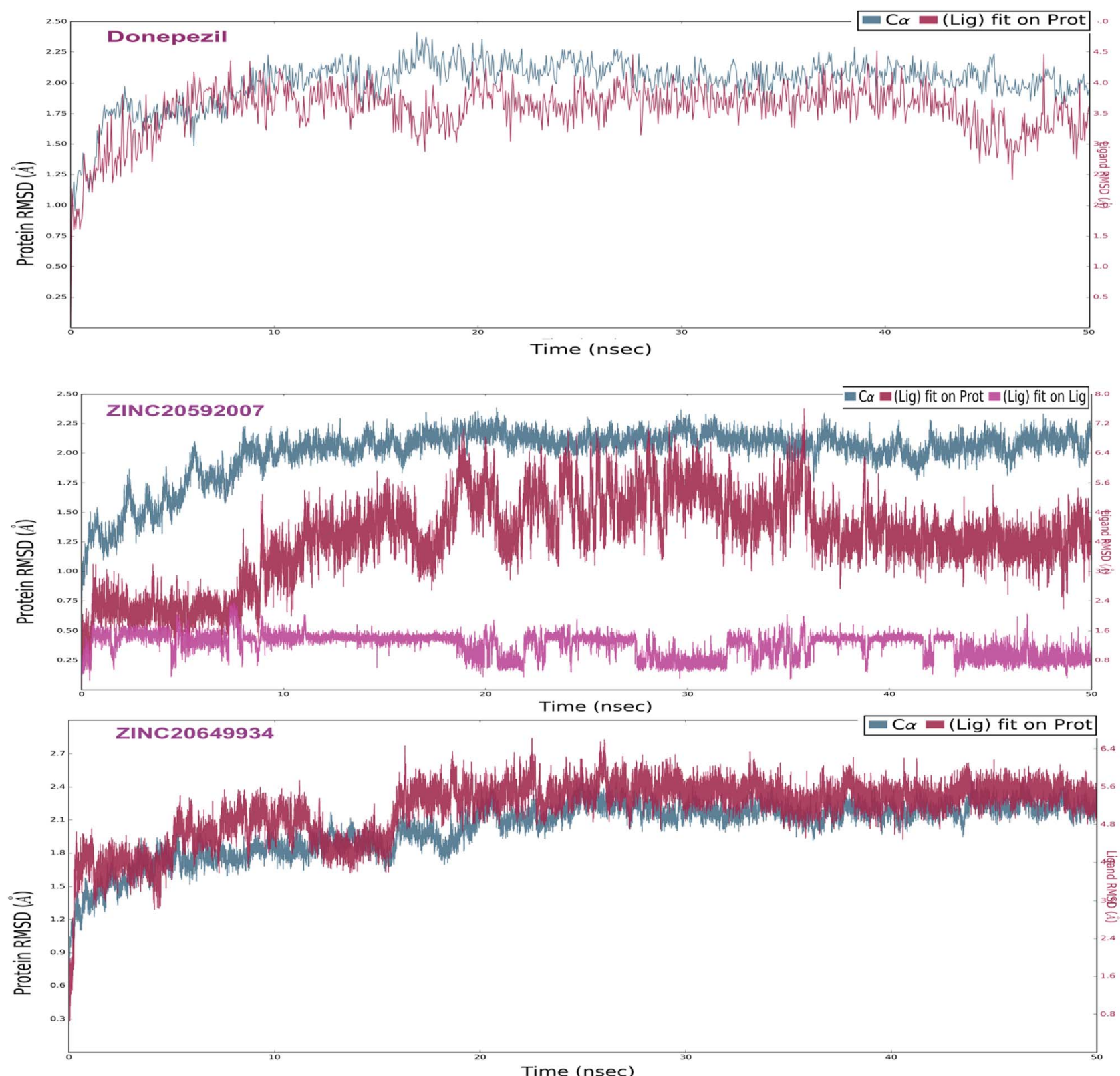

Fig. 11 RMSD plot (donepezil-AChE, ZINC20592007-AChE, and ZINC20649934-AChE complexes) of RMSD values for protein on the left $Y$ axis and for ligand these values were displayed on right $Y$-axis; protein backbone in green color, and ligand in maroon color. 
unaffected apparent value of $K_{\mathrm{m}}$, (ESI, Table $\mathrm{S} 4 \dagger$ ), indicated as non-competitive inhibitor on the kinetic constants. The double reciprocal Lineweaver-Burk displayed a nest of lines that intersect at a point other than $y$-axis, and intersecting lines converge to the left of $y$-axis, and below the $x$-axis, i.e., $\alpha<1$, and indicating that the inhibitor binds with greater affinity to the enzyme-substrate (ES) complex or subsequent species. The calculated inhibitor constant $\left(K_{\mathrm{i}}\right)$ of hits (ZINC20592007, ZINC05354646, ZINC20649934, and ZINC39154782) were $0.21 \pm$ $0.027 \mu \mathrm{M}, 0.27 \pm 0.064 \mu \mathrm{M}, 0.3 \pm 0.018 \mu \mathrm{M}$, and $0.28 \pm 0.032$ $\mu \mathrm{M}$ respectively and were attractive.

\section{Propidium iodide displacement assay}

The particular PAS site binding affinity through Trp286 amino acid residue was established by propidium iodide displacement method. The hits successfully displaced propidium, and were selective PAS ligands (Table 10). Molecule ZINC20592007 and ZINC39154782 displaced $100 \%$ propidium from PAS of AChE at $3 \mu \mathrm{M}$ concentration, but ZINC05354646 and ZINC20649934 displaced $57 \%$ and $58 \%$ respectively, at the same concentration.

\section{In vitro blood-brain barrier permeation assay}

A parallel artificial membrane permeation assay of bloodbrain barrier (PAMPA-BBB) was performed, as the method described by Di L. et al.,${ }^{27}$ to explore infiltration of the selected hits into brain. The in vitro permeability (Pe) of the four hits (Table 11) and nine commercially available drugs (Table S5, ESI $\dagger$ ) was determined through a lipid extract of porcine brain lipid in PBS. The assay was validated by comparing the experimentally obtained permeability $\left[\mathrm{Pe}_{(\exp )}\right]$ of the nine drugs with reported values of permeation $\left[\mathrm{Pe}_{(\mathrm{ref})}\right]$ offering a linear relationship, i.e., $\mathrm{Pe}_{(\exp )}=1.308 \mathrm{Pe}_{(\text {literature })}-0.8394$, $\left(R^{2}=0.9317\right)$.

The permeability values $(\mathrm{Pe})$ greater than $4.3 \times 10^{-6} \mathrm{~cm} \mathrm{~s}^{-1}$ were capable of CNS permeability (Fig. S8 \& Table S6, ESI $\dagger$ ) and the tested compounds demonstrated permeability values above it. Thus, the experimentally determined permeability values $(\mathrm{Pe})$ of the test compounds were a pointer towards their potential to comfortably cross the BBB by passive diffusion.
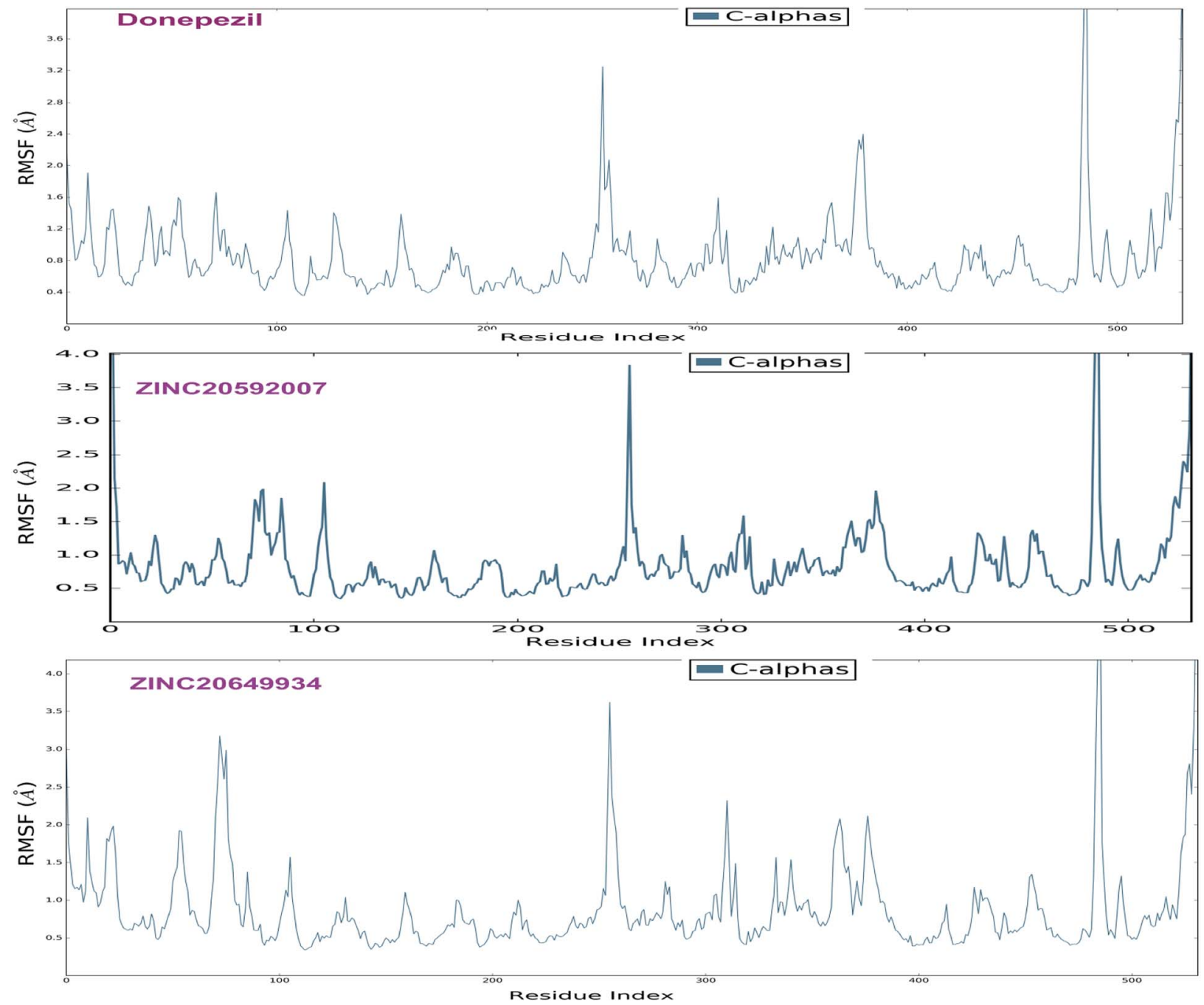

Fig. 12 RMSF of the protein C- $\alpha$ chain in donepezil-AChE, ZINC20592007-AChE, and ZINC20649934-AChE complexes. 

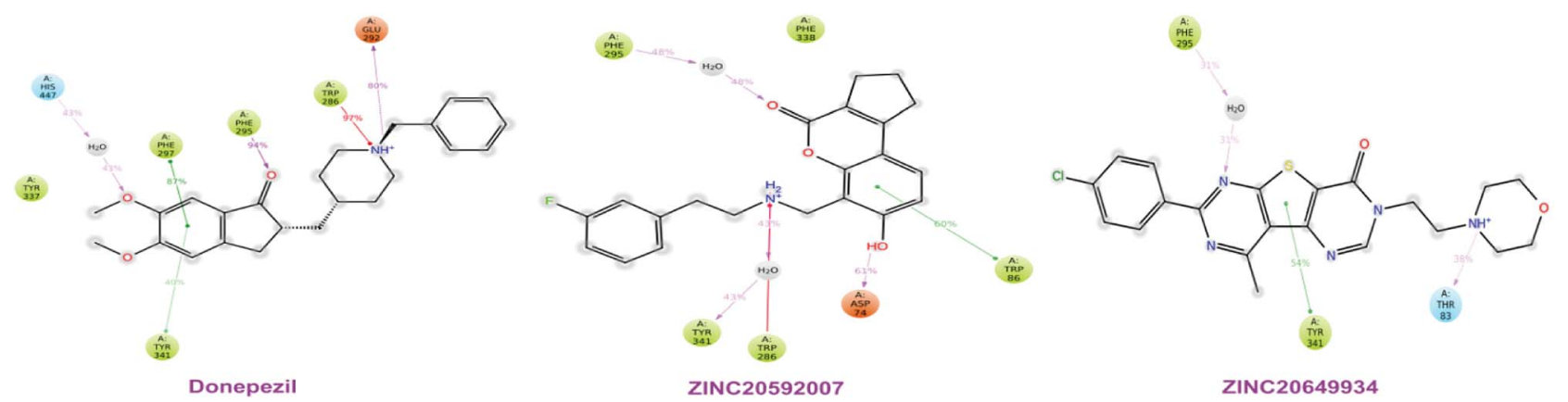

Fig. 13 Schematic diagram of detailed ligand (donepezil, ZINC20592007, and ZINC20649934) interactions with AChE protein amino acid residues after MD simulation.

\section{Cellular cytotoxicity and neuroprotection assessment}

The cell viability and neuroprotective potential, against apoptosis of selected hits, were evaluated by utilizing human neuroblastoma SH-SY5Y cell line. To investigate the cytotoxicity of compounds, cells were exposed to considerably high concentrations of the test compounds $(50 \mu \mathrm{M}$ and $100 \mu \mathrm{M})$ for $24 \mathrm{~h}$. The cell viability was determined by 3-(4,5-dimethyl thiazol-2-yl)-2,5-diphenyltetrazolium bromide (MTT) assay. The selected compounds showed insignificant cell death even at high concentrations (Table 12). The neuroprotective potential of selected hit molecules was assessed by using L-glutamate as excitotoxicity. In this assay, addition of L-glutamate $(100 \mu \mathrm{M})$ to growth media caused significant cell death as was evidenced by reduction in cell viability. The results (Table 12) are mean \pm SEM of at least three independent experiments.

\section{Molecular dynamics (MD) simulation}

The analyses of molecular dynamic (MD) simulation of ZINC20592007, ZINC20649934 and donepezil with AChE were performed to establish the binding potency and amino acid residue interactions. In MD simulations, RMSD of the protein backbone $\mathrm{C}-\alpha$ atoms and individual inhibitor, Root Mean Square Fluctuation (RMSF) in the individual amino acid side chain and ligand-AChE interactions were recorded concerning time over a period of $50 \mathrm{~ns}$ of simulation. The total energy of dynamic ligand-protein complexes was found stable in last 40 ns of entire simulation. Furthermore, temperature, pressure, volume, and potential energy of the complex remained constant, indicating the robustness and reliability of MD simulations. The RMSD of simulation converging between 1.5 and $2.5 \AA$, denoted the stability of macromolecular ligandprotein complexes during 50 ns simulation. The RMSF in individual amino acid residues during the entire simulation was below $4.0 \AA$, indicating a lower degree of conformational changes in the side chains.

After initial 10 ns simulation, RMSD of protein backbone C$\alpha$ along with the ligand RMSD values were stabilized. RMSD plot of RMSD values for protein on the left $Y$-axis and for ligand these values were displayed on right $Y$-axis in Fig. 11; protein backbone in green color, and ligand in maroon color. The mean RMSD value for donepezil-AChE complex was $2.04 \AA$, whereas
ZINC20592007-AChE and ZINC20649934-AChE complexes were 1.76 and $2.11 \AA$ respectively. RMSF was useful for characterizing local changes along the protein chain $\mathrm{C}-\alpha$ and peaks indicated areas of the protein that fluctuate the most during the simulation. RMSF values of hits and donepezil were below $4.0 \AA$, indicated less fluctuation and better stability of ligand-protein complex during simulation (Fig. 12). The interaction of hits with AChE enzyme higher than $30 \%$ after MD simulation is provided in Fig. 13.

MD study revealed that ZINC20649934 was interacted Phe 295 with H-bonding through a water molecule, Thr 83 residue with direct $\mathrm{H}$-bonding, and Tyr 341 amino acid with $\pi-\pi$ stacking; ZINC20592007 interacted Tyr341, Trp 286, and Phe 295 with H-bond formation through a water molecule, Trp86 with direct H-bonding, and Asp 74 with $\pi-\pi$ stacking; and donepezil interacted Trp286, Phe297, and Tyr241 with $\pi-\pi$ stacking, and Glu292, and Phe295 with H-bond, and His447 through water involvement $\mathrm{H}$-bonding. MD simulation displayed that all the ligands were interacting with protein at Phe 295 and Trp286, which were present at PAS site of AChE.

\section{Materials and methods}

\section{Computational details}

The computational tasks, except MD, were performed on an Intel(R) Core (TM) i5-3210M CPU @ $2.50 \mathrm{GHz}$ processor with a memory of 8.0 GB RAM running on a Linux 64 operating system. Schrödinger suite 2015-1 (Schrödinger, LLC, New York, NY, 2015) was utilized to develop structure-based and ligandbased pharmacophore models and for the screening of publicly free 'ZINC15' database. MD simulation was perform using Desmond package on an Intel(R) Xeon(R) CPU E31225v5@3.30 GHz $3.31 \mathrm{GHz}$ processor, RAM 32.0 GB system with Nvidia 'Quadro P600' GPU running on a Linux 64 operating system.

\section{Development of ligand-based pharmacophore}

Total 1062 structurally diverse AChE inhibitors with known and wide range of $\mathrm{IC}_{50}$ values $(0.043-20000 \mathrm{nM})$ were collected from Binding DB database (2017). The LigPrep in Maestro, Schrödinger 2015-1 was utilized to minimize the energy of inhibitor molecules by applying OPLS_2005 force field. ${ }^{28}$ As, 


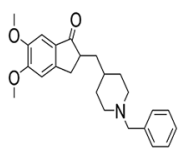

Donepezil, 4EY7,2.35 A Dihydrotanshinone I, 4M0E, 2.0 A

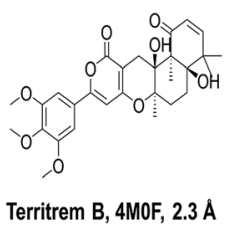

Fig. 14 Cocrystal ligands structure with PDB id and resolution. donepezil has mixed type (PAS and CAS site) of binding properties with AChE, therefore, all the compounds were clustered by Tanimoto similarities against donepezil using linear fingerprint descriptors with Canvas v2.3. Compounds were collected depending upon canvas similarity higher than and equal to 0.15 and molecular weight below 500. Finally, 142 inhibitors (Fig. S9 in ESI $\dagger$ ) were selected based on Glide docking study at PAS of AChE. The $\mathrm{IC}_{50}$ values of inhibitors were converted to $\mathrm{pIC}_{50}$ for the generation of 3D-QSAR model. PHASE v4.2, Schrödinger 2015-1 was used to generate 3D-QSAR model. ${ }^{29}$ The ConfGen, Schrödinger 2015-1 was used to create maximum 1000 number of conformers per structure utilizing force field OPLS_2005. The threshold of actives was above 8.0 and inactives was below 5.7. The PHASE randomly divided all ligands into two sets, i.e., the test set contained 42, and the training set included 100 compounds, to develop an Atom-based 3D-QSAR model in 1.00 $\AA$ of grid spacing. The ligands used for development of pharmacophore hypothesis are listed (ESI, Table S7†) with their fitness score, observed $\mathrm{pIC}_{50}$, phase predictive activity, and errors (the difference between observed and predicted activity). The common pharmacophore was obtained from the score of hypotheses having the best alignment of the active set ligands. All 142 compounds were aligned with the template pharmacophore hypothesis of the highly active molecule (Fig. S10 in ESI $\dagger$ ).

\section{Validation of ligand-based pharmacophore}

The QSAR model was developed with partial least-squares (PLS) factors one to five and was validated by predicting $\mathrm{pIC}_{50}$ value of molecules. The QSAR model with PLS factor 5 was considered as the best model. The 3D-QSAR models were externally validated by using LOO method to evaluate the predictivity of hypotheses. $^{30,31}$

\section{Energy-optimized structure-based pharmacophore generation}

Out of total 15, three X-ray crystal structures of hAChE were collected with good resolution and PAS site AChE inhibition activity of cocrystal ligand from the Protein Data Bank (https:// www.rcsb.org). The cocrystal ligands of three PDB structures viz. $4 \mathrm{EY7}$ (donepezil, $\mathrm{IC}_{50} 5.3 \mathrm{nM}$ ), 4M0E (dihydrotanshinone I, $K_{\mathrm{i}}$ $700 \mathrm{nM}$ ), and $4 \mathrm{M} 0 \mathrm{~F}$ (territrem B, IC $507 \mathrm{nM}, \& K_{\mathrm{i}} 1.7 \mathrm{nM}$ ) are shown in Fig. 14.

Protein structures were prepared using protein preparation wizard in Maestro 10.1, Schrödinger 2015-1 with an OPLS_2005 force field. The Grids of all three PDB structures were prepared at the center of cocrystal ligand using receptor Grid Generation tool in Maestro 10.1, Schrödinger 2015-1. The refined crystal ligands were docked by utilizing Glide XP (extra precision) docking with corresponding protein structures. The Glide XP energy was ranked by their contribution for the binding of pharmacophoric sites to cocrystal ligand. ${ }^{32}$ PHASE v4.2, Schrödinger, 2015-1 was applied to generate pharmacophore features based on XP energy descriptor information. It was used to develop pharmacophore sites viz. H-bond acceptor (A), $\mathrm{H}$ bond donor (D), hydrophobic group (H), negative ionizable group $(\mathrm{N})$, positive ionizable group $(\mathrm{P})$, and aromatic ring $(\mathrm{R}) . \mathrm{H}-$ bond acceptor and $\mathrm{H}$-bond donor were pointed as vectors, directed to corresponding $\mathrm{H}$-bond donor and acceptor positions at the binding site of receptors respectively. The Glide XP descriptors consisted of hydrophobic enclosure, hydrophobically packed associated hydrogen bonds, electrostatic rewards, $\pi-\pi$ stacking, $\pi$-cation, and other interactions. The most favorable sites were selected for the development of epharmacophore hypothesis by using excluded volume.

\section{Energy-optimized structure-based pharmacophore validation}

Enrichment factor (EF) and goodness of hit (GH) were calculated to validate e-pharmacophore hypotheses (eqn (1) and (2) respectively). A dataset of compounds was prepared using 1000 drug-like decoys (http://www.schrödinger.com/glide_decoy_set) with an average molecular weight of $400 \mathrm{D}$ (the "dl-400" dataset) and known actives of $53 \mathrm{AChE}$ inhibitors (inhibitors with $\mathrm{IC}_{50}$ less than $100 \mathrm{nM}$ and out of molecules utilized for the 3D-QSAR model), to validate e-pharmacophore models. LigPrep in Schrödinger 2015-1 with Epik was applied to prepare database ligands with an OPLS_2005 force field. EF is the fraction of known actives retrieved after a screening of decoy database compounds. $^{33}$

$$
\begin{gathered}
\mathrm{EF}=\frac{\mathrm{Ha} \times D}{\mathrm{Ht} \times A} \\
\mathrm{GH}=\left[\frac{(\mathrm{Ha}(3 A+\mathrm{Ht}))}{4 \mathrm{Ht} A}\right]\left[1-\frac{(\mathrm{Ht}-\mathrm{Ha})}{(D-A)}\right]
\end{gathered}
$$

where, $\mathrm{EF}=$ enrichment factor, $\mathrm{GH}=$ goodness of hit, $D=$ total compounds in the data set, $A=$ total number of actives in the data set, $\mathrm{Ht}=$ total hits, and $\mathrm{Ha}=$ active hits.

\section{Pharmacophore-based screening of the database}

Only 'hit-like' compounds without known AChE inhibitors were collected from 'ZINC15' database utilizing Lipinski's filter. LigPrep with Epik was employed to prepare database ligands utilizing OPLS_2005 force field. One ligand-based pharmacophore and three e-pharmacophores based matched molecules were separately screened against prepared database compounds with PHASE v4.2, Schrödinger 2015-1. ${ }^{34}$ Pharmacophore matching was required for the most energetically favorable site, and score of more than $1.0 \mathrm{kcal} \mathrm{mol}^{-1}$ was selected for the pharmacophore screening, four sites for hypotheses with 3 or 4 and five sites for hypotheses with 4 or 5 were required to match. The tolerance of distance matching was set up to $2.0 \AA$ A. The aligned conformer of molecule matches the hypothesis based on rmsd, site matching, vector alignments, 
and volume terms expressed as fitness score..$^{35}$ The pharmacophore matched database was ranked in order of the fitness score ranging from 0 to 3 , as applied in the PHASE. The ligands were selected based on highest fitness scores up to 2000 molecules for each pharmacophore and scores above 1.5 were considered as suitable inhibitors. The molecules with best fitness score were docked into the binding sites of AChE crystal structure. $^{32}$

\section{Removal of pan-assay interference compounds (PAINS)}

Baell and Holloway reported a list of structural features which generated frequent false positives across screening, known as PAINS. ${ }^{24}$ Jasial S. et al. established a large-scale analysis of behavior of PAINS in biological screening assays. ${ }^{36}$ The 'ZINC15' database molecules are categories within (A) anodyne, and (B) clean (PAINS-ok), ${ }^{37}$ were selected as hits from HTVS retrieves. A KNIME (freely available Konstanz Information Miner, http:// knime.org) ${ }^{38}$ workflow distributed with the RDKit ${ }^{39}$ software package utilizing GUI data analysis platform was developed by Saubern S. et al. ${ }^{40}$ The obtained HTVS hits were screened in silico for PAINS to avoid false positives in biochemical and pharmacological assays using three public filters, including RDKit, ${ }^{39}$ ZINC, $^{37}$ and FAF-Drugs4 server. ${ }^{41}$

\section{High throughput virtual screening (HTVS) and molecular docking}

Glide HTVS (high throughput virtual screening) is faster than Glide SP and XP, has higher tolerance to suboptimal fits than Glide XP and thus is selected for the study. ${ }^{33}$ After removal of PAINS, e-pharmacophore matched compounds were docked into binding sites of respective crystal structures, and ligandbased matched molecules were docked into 4M0E structure with Glide, Schrödinger 2015-1. ${ }^{42}$ The grid was generated at the center position of cocrystal ligand, through Grid Generation tools in Glide. Post-docking MM-GBSA minimization was performed to optimize the ligand geometries. The Glide HTVS screened molecules with best docking scores were selected for Glide SP (standard precision), and XP (extra precision) screenings. Top $10 \%$ of retrieves out from each step were taken up for next step. Finally, all the non-peptide retrieves from HTVS and donepezil were docked in Glide XP molecular docking using $4 \mathrm{M} 0 \mathrm{E}$ crystal structure (highest resolution PDB with cocrystal ligand, $2.0 \AA$ ) to compare the docking score of screened out retrieves with reference donepezil.

\section{Induced fit docking}

We applied a mixed molecular docking and dynamics method known as induced fit docking (IFD), ${ }^{43}$ where the receptor was flexible in docking study. After ADME screening, selected hits were prepared by OPLS_2005 force field utilizing LigPrep. The hits were docked to rigid protein by using Glide, Schrödinger 2015-1 with scaling of ligand van der Waals (vdW) radii 0.5 for nonpolar atoms. ${ }^{\mathbf{4 4}}$ Constrained energy minimization was performed on AChE (PDB: 4M0E) crystal structure, keeping it close to the original crystal structure while removing bad steric contacts. The energy minimization of protein structure was performed using OPLS_2005 force-field. The Glide XP was utilized for initial docking, and 20 ligand poses were retained for protein structural refinement. Prime, Schrödinger 2015-1 was used to refine residue within $5.0 \AA$ of ligand poses and to generate the induced-fit protein-ligand complexes. Each of the 20 complexes was subjected to refinements of side-chain and backbone, ${ }^{44}$ and were ranked according to Prime energy. The receptor structures within $30 \mathrm{kcal} \mathrm{mol}^{-1}$ were redocked for final round of Glide docking and scoring. The Prime refinement included at least one atom of all residue located within $4.0 \AA$ of corresponding ligand pose. In the last step, every ligand was redocked into each refined low-energy receptor structure generated in the refinement step. The new 20 receptor conformations were taken forward for Glide XP redocking. The binding affinity of each complex was reported in the docking score. The more negative docking score indicates more favorable binding with receptor.

\section{Prime MM-GBSA simulation}

The free binding energies of highest scoring docked complexes were computed utilizing molecular mechanic-generalized Born surface area (MM-GBSA) ${ }^{\mathbf{4 5}}$ followed by default parameters. Based on the docking score and MM/GBSA binding-free energy, Jin et al. developed correlation model between docking scores or calculated binding-free energies and experimental $\mathrm{pIC}_{50}$ values. ${ }^{46}$ The Prime (Maestro v10.1, Schrödinger, LLC, New York, NY, 2015) was employed to calculate the MM-GBSA energy of Glide XP docked complex. The OPLS_2005 force field in conjunction with GBSA continuum $\operatorname{model}^{47}$ was utilized to determine energies of selected complexes of ligands. Computationally, the binding free energies $\left(\Delta G_{\text {bind }}\right)$ of ligands were calculated using the following equation. ${ }^{48}$

$$
\Delta G_{\mathrm{bind}}=\Delta E_{\mathrm{MM}}+\Delta G_{\mathrm{solv}}+\Delta G_{\mathrm{SA}}
$$

where $\Delta E_{\mathrm{MM}}$ is the difference between minimized energies of the AChE-inhibitor complex and sum of the minimized energies of unliganded AChE and its inhibitor, $\Delta G_{\text {solv }}$ is difference between GBSA solvation energies of enzyme-inhibitor complex and sum of the GBSA solvation energies of unliganded AChE and inhibitor, and $\Delta G_{\mathrm{SA}}$ is the difference between surface area energies of the complex and sum of the surface area of unliganded enzyme and its inhibitor.

\section{Docking using Autodock}

AutoDockTools-1.5.6. and AutoDock 4.2 suite were utilized to redock the selected hits as AChE inhibitor for comparison of the Glide XP docking, IFD, and AutoDock results. AChE crystal structure, 4M0E, was prepared using AutoDock Tools. Atom charges, solvation parameters, and polar hydrogens were added to enzyme structure for docking simulation before applying to PDBQT file format. The Chem3D 16.0 chemical structure drawing software was utilized to draw hits with standard 3D structures and to minimize energies of the compounds using MM2 energy minimization method. ${ }^{49}$ The AutoDock 4.2 ligand optimization was performed using Gasteiger charges 
optimization, non-polar hydrogens were merged, and saved as PDBQT file. AutoDock requires pre-calculated grid maps, and the grid must surround the region of active site of AChE. Therefore, the grid box was centered at the active site including Leu 289, Arg 296, Phe 297, Phe 338, Trp 286, Ser 293, Val 294, Phe 295, Tyr 72, Tyr 341, Asp 74, Tyr 124 and Tyr 337 amino acid residues. The grid box size was positioned at 40,42 , and $48 \AA$ and the grid center was set to $20.683,-16.615,19.006$ for $x, y$ and $z$ respectively, covering the active pocket. AutoGrid 4.0 was used to produce a grid with $0.375 \AA$ spacing between grid points. The Lamarckian Genetic Algorithm (LGA) was used to search best conformers, and a maximum of 50 conformers was considered for each compound with the default setting. The Discovery Studio Visualizer was used for visualization of interactions. AutoDock Tools provided various methods for analyzing the results of docking simulations, viz. conformational similarity, visualizing the binding site and its energy, intermolecular energy and inhibition constant.

\section{ADME properties prediction}

The QikProp in Maestro 10.1, Schrödinger 2015-1 (ref. 50) was used to predict ADME properties of hit molecules. As the QikProp was unsuitable to neutralize the compounds and generate the descriptors, in the normal mode, hence, neutralization of all molecules was essential before performing QikProp. The QikProp predicted physicochemically significant and pharmaceutically applicable 44 descriptors for the hits. These included principle descriptors, physiochemical properties as well as $\log P$ (octanol/water), QP\%, log HERG, Caco-2 cell membrane permeability, MDCK cell permeability, skin permeability $\log K_{\mathrm{p}}$ and Lipinski's rule of five, which were crucial for rational drug design. ${ }^{51,52}$

\section{Density functional theory}

Density functional theory (DFT) is utilized to determine and validate enzymatic reaction mechanisms and the enzyme active sites. Electronic effects of drug-like compounds play an essential role in the pharmacological effects. ${ }^{53}$ The most and least active AChE inhibitors of training set were optimized with the final hits in Jaguar (Jaguar v8.7, Schrödinger, LLC, New York, NY, 2015) program utilizing Lee-Yang-Parr correlation functional (B3LYP) theory, and Becke's three-parameter exchange potential $^{54,55}$ with $6-31 \mathrm{G}^{*}$ basis set. The molecular orbital surfaces, atomic electrostatics potential charges (EPS) and molecular electrostatic potential (MESP) were determined to calculate the HOMO and LUMO. The HOMO energy of small ligand molecules can donate electrons during the drug-enzyme complex formation, while LUMO energy manifests the capacity of the molecule to accept the electrons from the protein. The HOMO-LUMO gap energy (difference in HOMO and LUMO energy), expresses the electronic excitation energy, that is essential to compute the molecular reactivity and stability of the drug-protein complex. ${ }^{25}$

\section{In silico AChE selectivity study}

To determine binding affinity of hits towards BuChE, we carried out XP docking of hits using crystal structure of 4BDS (highest resolution PDB of human BuChE, 2.1 $\AA$ ). The Glide (Glide, Schrödinger, LLC, New York, NY, 2015) was used to perform Glide XP docking in default setting for all docking steps, and the Grid was centralized at the PAS site of BuChE i.e., centralized the residues ASp70, Trp 82, Asn83, Ser198, and Tyr332. ${ }^{56}$

\section{In vitro $\mathrm{AChE}$ and BuChE enzyme inhibition}

The AChE and BuChE inhibition studies were performed by Ellman et al. method. ${ }^{26}$ Four selected hit molecules (ZINC20592007, ZINC05354646, ZINC20649934, and ZINC39154782) out of ten, were procured from MolPort SIA, Riga, Latvia (MolPort id: MolPort-002-672-705, MolPort-002658-497, MolPort-005-915-644, and MolPort-004-876-009 respectively). The AChE from Electrophorus electricus and BuChE from horse serum (lyophilized powder) (CAS No. 900081-1, CAS No. 9001-08-5, respectively) were purchased from Sigma Aldrich, India. Acetylthiocholine iodide (ATCI), butyrylthiocholine iodide (BTCI), 5,5'-dithio-bis(2-nitrobenzoic acid) (DTNB-Ellman's reagent) and phosphate buffer saline (PBS), pH 7.4 were procured from HiMedia Laboratories, India, and donepezil (Sigma Aldrich, India) was used as reference. Six different concentrations (75 $\mu \mathrm{M}, 15 \mu \mathrm{M}, 7.5 \mu \mathrm{M}$, $3 \mu \mathrm{M}, 0.6 \mu \mathrm{M}$, and $0.12 \mu \mathrm{M}$ ) of hits, $0.25 \mathrm{mM}$ DTNB, 0.06unit $\mathrm{mL}^{-1}$ of AChE or BuChE were combined in PBS and incubated at $37^{\circ} \mathrm{C}$ for $30 \mathrm{~min}$ to determine inhibition of AChE or BuChE. $0.36 \mathrm{mM}$ of the substrate (ATCI or BTCI) was added to reaction mixture before measuring absorbance at $415 \mathrm{~nm}$ wavelength by Synergy HTX multi-mode reader (BioTek, USA). The process was performed in triplicate with a blank and control, to calculate the percentage inhibition due to selected hits. The $\mathrm{IC}_{50}$ values, i.e., the concentration of the drug resulting in $50 \%$ inhibition of enzyme activity, were determined graphically from inhibition curves (log inhibitor concentration $v s$. percent inhibition) utilizing GraphPad Prism 5.0, GraphPad Software Inc. ${ }^{57}$

The enzyme kinetics (the mechanism of inhibition by ligands) of were determined by previously described method. ${ }^{26}$ Eight concentrations of substrate (ATCI; 0.1-1.15 $\mu \mathrm{M}$ ) were incubated with AChE in absence and presence of different concentrations of test molecules $(0.25 \mu \mathrm{M}, 0.5 \mu \mathrm{M} \& 1 \mu \mathrm{M}$ for ZINC20592007, and ZINC05354646; and $0.5 \mu \mathrm{M}, 1 \mu \mathrm{M} \& 2 \mu \mathrm{M}$ for ZINC20649934, and ZINC39154782). The absorbance was measured for $30 \mathrm{~min}$ at intervals of $5 \mathrm{~min}$ at $415 \mathrm{~nm}$ wavelength. The products formed during the time frame of $30 \mathrm{~min}$ were estimated by Beer-Lambert law. $V_{\max }$ and $K_{\mathrm{m}}$ values of Michaelis-Menten kinetics were computed by nonlinear regression from substrate-velocity curves using GraphPad Prism 5. Linear regression was used to calculate inhibition constant $\left(K_{\mathrm{i}}\right)$ utilizing Lineweaver-Burk plots. ${ }^{58} K_{\mathrm{i}}$ value was determined by Yonetani-Theorell method in which the lines from the double reciprocal Lineweaver-Burk plot were extrapolated to intersect at a point. ${ }^{59}$ The positive reciprocal $x$-values of intersecting point were the determined $K_{\mathrm{i}}$ value 
of hits. The enzyme kinetics assays were performed in triplicate.

\section{Assay of propidium iodide displacement}

The molecular modeling studies illustrated that selected hits were PAS selective AChE inhibitors. Propidium iodide is a specific PAS selective ligand, which displays 10-fold fluorescence enrichment when bound to AChE. The displacement by hits was measure of their affinity towards PAS of AChE. Three concentrations $(0.24,1.0$, and $3.0 \mu \mathrm{M})$ of test compounds, $5 \mu \mathrm{M}$ AChE from electric eel (eeAChE) in PBS, pH 7.4, were added in black 96-well plates and were kept at room temperature for $6 \mathrm{~h}^{60}$ The sample solutions were incubated for $15 \mathrm{~min}$ with 20 $\mu \mathrm{M}$ of propidium iodide (HiMedia, India), and intensity of fluorescence was measured in excitation and emission modes at 485 and $620 \mathrm{~nm}$, respectively. The assay was carried out in triplicate.

\section{In vitro blood-brain barrier permeation assay}

The possible in vitro blood-brain barrier (BBB) permeation of compounds was predicted by parallel artificial membrane permeation assay (PAMPA) of BBB as described by Di L. et al. ${ }^{27}$ The donor microplates (PVDF membrane, pore size $0.45 \mu \mathrm{m}$ ) and acceptor microplates were obtained from Millipore, Bengaluru, India. The filter surface of donor microplate was impregnated with $4 \mu \mathrm{L}$ of $20 \mathrm{mg} \mathrm{mL}^{-1}$ porcine brain lipid (Avanti polar lipids, Alabaster) in dodecane (Avra Synthesis, Hyderabad, India), and the acceptor microplates were filled with $200 \mu \mathrm{L}$ of PBS, pH 7.4. $5 \mathrm{mg} \mathrm{mL}^{-1}$ of test compounds were dissolved in DMSO and diluted with PBS to obtain a final concentration of $100 \mu \mathrm{g} \mathrm{mL} \mathrm{m}^{-1}$. The donor well plates were filled with $200 \mu \mathrm{L}$ of test solution and were carefully placed on the acceptor plate like a sandwich, carrying it undisturbed for $18 \mathrm{~h}$ at $25{ }^{\circ} \mathrm{C}$. The donor plates were then removed, and concentration of compounds in acceptor, and donor wells were determined by measuring absorbance. Each well was analyzed at five different wavelengths with three independent performances, and results were explicit as mean \pm SEM. The nine commercial drugs with known BBB permeability (verapamil, diazepam, progesterone, atenolol, dopamine, lomefloxacin, alprazolam, chlorpromazine, and oxazepam) were utilized to validate PAMPA model. The above-described method was followed to determine the experimental permeability, $\mathrm{Pe}_{(\exp )}$ values of these drugs, and data were regressed against $\mathrm{Pe}_{(\mathrm{ref})}$ from literature to establish a linear correlation. ${ }^{61}$

\section{Determination of cellular cytotoxicity and neuroprotection}

Neuronal cell line cultures. The human neuroblastoma $\mathrm{SH}$ SY5Y cell line was procured from National Centre for Cell Science (NCCS) Pune, India. Cells were cultured into T25 flasks containing Dulbecco's modified Eagle's medium nutrient mixture F-12 (DMEM-F12), supplemented with 10\% fetal bovine serum (FBS), $1 \mu \mathrm{M}$ glutamine, $50 \mathrm{U} \mathrm{mL}^{-1}$ penicillin, and $50 \mu \mathrm{g}$ $\mathrm{mL}^{-1}$ streptomycin and were maintained at $37{ }^{\circ} \mathrm{C}$ in $5 \% \mathrm{CO}_{2}$ humidified air. For MTT assay and neuroprotection study, $\mathrm{SH}-$
SY5Y cells were subcultured in 96-well plates at seeding density of $5 \times 10^{4}$ cells per well.

Determination of cell viability and neuroprotection. The MTT (3-(4,5-dimethyl thiazol-2-yl)-2,5-diphenyltetrazolium bromide) assay $^{62}$ was performed to determine cytotoxicity of selected hits. After $24 \mathrm{~h}$ incubation at $37^{\circ} \mathrm{C}$, the medium was changed with test compounds having concentrations of $50 \mu \mathrm{M}$ and $100 \mu \mathrm{M}$, for another $24 \mathrm{~h}$ at previously described conditions. $5 \mathrm{mg} \mathrm{mL} \mathrm{mL}^{-1}$ of MTT (Sigma-Aldrich, India) in PBS was added to the culture medium for $4 \mathrm{~h}$ at $37^{\circ} \mathrm{C}$. The medium was removed, and the blue formazan crystals formed were dissolved in DMSO and evaluated by measuring absorbance at $570 \mathrm{~nm}$. The test was carried out in triplicate, and results were explicit as mean \pm SEM.

Neuroprotectivity of selected hits was determined by evaluating their ability to protect SH-SY5Y cells against induced apoptosis by L-glutamate excitotoxicity. Amyloid beta $(\mathrm{A} \beta)$ neurotoxicity was triggered by L-glutamate in SH-SY5Y cell line. ${ }^{63}$ The cells were treated with test compounds, at $25 \mu \mathrm{M}$ concentration, and incubated for $2 \mathrm{~h}$. After incubation, cells were treated with a medium containing $100 \mu \mathrm{M}$ of L-glutamate and left for an additional $24 \mathrm{~h}$. The cell viability, after the treatment of L-glutamate, was assessed by MTT assay. The medium was further replaced with $80 \mu \mathrm{L}$ of fresh medium and $20 \mu \mathrm{L}$ of MTT $\left(0.5 \mathrm{mg} \mathrm{mL} \mathrm{mL}^{-1}\right)$ in PBS. After $4 \mathrm{~h}$ of incubation, MTT solution was removed, and the crystals of formazan were dissolved in DMSO to measure the absorbance at $570 \mathrm{~nm}$. Percentage of neuronal cell protection against L-glutamate was calculated by considering the absorbance of the control cells as $100 \%$ of the cell viability.

\section{Molecular dynamics (MD) simulation}

MD simulations of ZINC20592007 (most active in in vitro tests and 100\% PAS selective), ZINC20649934 (higher docking score in in silico and 58\% PAS selective in in vitro), and donepezil were performed utilizing Desmond v2.2, Schrödinger 2015-1 with the OPLS 2005 force field to model all peptide interactions, ${ }^{64,65}$ and TIP3P (transferable intermolecular potential with 3 points) model was used for solvent. Protein-ligand docked complex (.pv file) from XP docking was taken for solvation using open TIP3P water model in an orthorhombic core box of $20 \AA$ radius. The overall complex had six negative charges and was neutralized by adding $\mathrm{Na}^{+}$counter ion for simulation. Ligand-protein complex was minimized by steepest descent method followed by BFGS (Broyden-FletcherGoldfarb-Shanno) algorithm having a convergence threshold of $2.0 \mathrm{kcal} \mathrm{mol}^{-1}$ and 41667 iterations. Ewald method (PME) ${ }^{66}$ was used to calculate long-range electrostatic interactions with a grid spacing of 0.8. van der Waals and short-range electrostatic interactions were truncated at 9.0. Nose-Hoover thermostats $^{67}$ were utilized to maintain constant simulation temperature, and Martina-Tobias-Klein method ${ }^{68}$ was used to control pressure throughout simulation. The equations of motion were integrated utilizing the multistep RESPA integrator $^{69}$ with an internal time step of 2.0 fs for bonded interactions and non-bonded interactions within 6.0 fs cut off. MD 
simulations were conceded out at $300 \mathrm{~K}$ temperature and 1.01325 bar pressure. The overall model system was relaxed for $2 \mathrm{~ns}$ before a $50 \mathrm{~ns}$ simulation, and coulombic interactions were defined by a short-range cut off radius of $9.0 \AA$ and by a long-range smooth particle mesh Ewald tolerance to $1 \times$ $10^{-9}$. Further, for energy calculation and trajectory analysis, recording interval of $1.2 \mathrm{ps}$ was defined.

\section{Conclusions}

A 3D-QSAR and three e-pharmacophore models were developed from known AChE inhibitors, structurally similar to donepezil and available AChE crystal structures with cocrystal ligand at PAS site. Virtual screening of ZINC15 compounds afforded new excellent, non-toxic AChE inhibitors. The hits interacted with Trp 286, Phe 295, Asp 74, Tyr 337, and Tyr 124 residues of AChE crystal structure through one to three $\mathrm{H}$-bond(s) and one to three pi-pi stacking interaction(s). MD strongly supported that the identified hits bound at PAS of AChE only. In vitro enzyme assays, with propidium iodide displacement of ZINC20592007, ZINC05354646, ZINC20649934, and ZINC39154782, also supported the in-silico results. ZINC20592007 and ZINC39154782, interacting with Try 286 amino acid residue, provided $100 \%$ propidium displacement at $3 \mu \mathrm{M}$ concentration. The PAS siteselective mimics responded to inhibition of amyloid formation. The hits had attractive $K_{\mathrm{i}}$ values $(0.21 \pm 0.027 \mu \mathrm{M}, 0.27 \pm$ $0.064 \mu \mathrm{M}, 0.3 \pm 0.018 \mu \mathrm{M}$, and $0.28 \pm 0.032 \mu \mathrm{M})$ with insignificant toxicity against neuroblastoma SH-SY5Y cell, good BBB permeability, and neuroprotectivity against L-glutamate induced excitotoxicity.

Further, ZINC20592007 molecule had potent, selective AChE inhibition at PAS, i.e., non-competitive, CNS permeability, nontoxicity, neuroprotectivity, and $A \beta$ formation and aggregation inhibition, which increased cholinergic activity and also prevented $A \beta$ aggregation to control $A D$. We consider that these compounds are excellent candidates to develop further as leads for AChE inhibition.

\section{Conflicts of interest}

The authors declare no competing financial interest.

\section{Abbreviations}

$\begin{array}{ll}\text { AChE } & \text { Acetylcholinesterase } \\ \text { AChEI } & \text { AChE inhibitor } \\ \text { AD } & \text { Alzheimer's disease } \\ \text { ADME } & \begin{array}{l}\text { Absorption distribution metabolism and } \\ \text { excretion }\end{array} \\ \text { APP } & \text { Amyloid precursor protein } \\ \text { BBB } & \text { Blood brain barrier } \\ \text { BuChE } & \text { Butyrylcholinesterase } \\ \text { CNS } & \text { Central nervous system } \\ \text { 3D-QSAR } & \text { 3-Dimentional-quantitative structure activity } \\ & \text { relationship } \\ \text { EF } & \text { Enrichment factor }\end{array}$

e- $\quad$ Energy-optimized pharmacophore

pharmacophore

Glide XP Glide extra precision

GH Goodness of hit

HTVS High throughput virtual screening

IFD Induced fit docking

MD Molecular dynamics

MTT 3-(4,5-Dimethylthiazol-2-yl)-2,5-

diphenyltetrazolium bromide

OPLS Optimized potential for liquid simulations

PAINS Pan assay interference compounds

PAMPA Parallel artificial membrane permeation assay

RMSD Root mean square deviation

SP Standard precision

THA Tacrine

1YL (1R)-1,6-Dimethyl-1,2-dihydrophenanthro[1,2b]furan-10,11-dione

\section{Acknowledgements}

The authors are thankful to Department of Biotechnology, Ministry of Science \& Technology, New Delhi, India for financial support (BT/PR9624/MED/30/1253/2013 dated-29/11/2014). We are grateful to Dr Ozair Alam, Department of Pharmaceutical Chemistry, School of Pharmaceutical Education \& Research, Jamia Hamdard, Hamdard Nagar, New Delhi, India, for his assistance and support. S. J. and A. G. would like to thank Ministry of Human Resource Development, New Delhi, India, for the award of senior research fellowships to them.

\section{References}

1 Y. Bourne, P. Taylor, P. E. Bougis and P. Marchot, J. Biol. Chem., 1999, 274, 2963-2970.

2 G. Johnson and S. Moore, Curr. Pharm. Des., 2006, 12, 217225.

3 H. Soreq and S. Seidman, Nat. Rev. Neurosci., 2001, 2, 294302.

4 M. Pakaski and P. Kasa, Curr. Drug Targets: CNS Neurol. Disord., 2003, 2, 163-171.

5 N. P. L. Verhoeff, Expert Rev. Neurother., 2005, 5, 277-284.

6 J. Massoulié, J. Sussman, S. Bon and I. Silman, Prog. Brain Res., 1993, 98, 139-146.

7 J. Massoulie and S. Bon, Annu. Rev. Neurosci., 1982, 5, 57-106. 8 E. O. Campos, A. Alvarez and N. C. Inestrosa, Neurochem. Res., 1998, 23, 135-140.

9 N. C. Inestrosa, A. Alvarez, C. A. Perez, R. D. Moreno, M. Vicente, C. Linker, O. I. Casanueva, C. Soto and J. Garrido, Neuron, 1996, 16, 881-891.

10 M.-S. García-Ayllón, D. H. Small, J. Avila and J. Sáez-Valero, Front. Mol. Neurosci., 2011, 4, 22.

11 F. Ferreri, C. Agbokou and S. Gauthier, J. Psychiatry Neurosci., 2006, 31, 369.

12 S. R. Ribeiz, D. P. Bassitt, J. A. Arrais, R. Avila, D. C. Steffens and C. M. Bottino, CNS Drugs, 2010, 24, 303-317.

13 A. A. Kehagia, R. A. Barker and T. W. Robbins, Lancet Neurol., 2010, 9, 1200-1213. 
14 T. Brenner, Y. Hamra-Amitay, T. Evron, N. Boneva, S. Seidman and H. Soreq, FASEB J., 2003, 17, 214-222.

15 T. Brenner, E. Nizri, M. Irony-Tur-Sinai, Y. Hamra-Amitay and I. Wirguin, J. Neuroimmunol., 2008, 201, 121-127.

16 E. Giacobini, Int. J. Geriatr. Psychiatry, 2003, 18, S1-S5.

17 S. Jana and S. K. Singh, J. Biomol. Struct. Dyn., 2018, 1-22.

18 J. K. Dhanjal, S. Sharma, A. Grover and A. Das, Biomed. Pharmacother., 2015, 71, 146-152.

19 P. Ambure, S. Kar and K. Roy, BioSystems, 2014, 116, 10-20.

20 G. Brahmachari, C. Choo, P. Ambure and K. Roy, Bioorg. Med. Chem., 2015, 23, 4567-4575.

21 Y. Zhang, S. Zhang, G. Xu, H. Yan, Y. Pu and Z. Zuo, Mol. BioSyst., 2016, 12, 3734-3742.

22 S. Bag, R. Tulsan, A. Sood, S. Datta and M. Torok, Curr. Comput.-Aided Drug Des., 2013, 9, 2-14.

23 R. Malik, B. S. Choudhary, S. Srivastava, P. Mehta and M. Sharma, J. Biomol. Struct. Dyn., 2016, 1-17.

24 J. B. Baell and G. A. Holloway, J. Med. Chem., 2010, 53, 27192740.

25 Y. Zheng, M. Zheng, X. Ling, Y. Liu, Y. Xue, L. An, N. Gu and M. Jin, Bioorg. Med. Chem. Lett., 2013, 23, 3523-3530.

26 G. L. Ellman, K. D. Courtney, V. Andres Jr and R. M. Featherstone, Biochem. Pharmacol., 1961, 7, 88-95.

27 L. Di, E. H. Kerns, K. Fan, O. J. McConnell and G. T. Carter, Eur. J. Med. Chem., 2003, 38, 223-232.

28 Schrödinger, LLC, S. Release, New York, NY, 2015.

29 S. L. Dixon, A. M. Smondyrev and S. N. Rao, Chem. Biol. Drug Des., 2006, 67, 370-372.

30 P. Pratim Roy, S. Paul, I. Mitra and K. Roy, Molecules, 2009, 14, 1660-1701.

31 K. Loving, N. K. Salam and W. Sherman, J. Comput.-Aided Mol. Des., 2009, 23, 541-554.

32 N. K. Salam, R. Nuti and W. Sherman, J. Chem. Inf. Model., 2009, 49, 2356-2368.

33 T. A. Halgren, R. B. Murphy, R. A. Friesner, H. S. Beard, L. L. Frye, W. T. Pollard and J. L. Banks, J. Med. Chem., 2004, 47, 1750-1759.

34 J. J. Irwin, T. Sterling, M. M. Mysinger, E. S. Bolstad and R. G. Coleman, J. Chem. Inf. Model., 2012, 52, 1757-1768.

35 S. L. Dixon, A. M. Smondyrev, E. H. Knoll, S. N. Rao, D. E. Shaw and R. A. Friesner, J. Comput.-Aided Mol. Des., 2006, 20, 647-671.

36 S. Jasial, Y. Hu and J. r. Bajorath, J. Med. Chem., 2017, 60, 3879-3886.

37 T. Sterling and J. J. Irwin, J. Chem. Inf. Model., 2015, 55, 23242337.

38 M. R. Berthold, N. Cebron, F. Dill, T. Gabriel, T. Kötter, T. Meinl, P. Ohl, C. Sieb, K. Thiel and B. Wiswedel, Data Analysis, and Knowledge Organisation (GfKL 2007), Springer, 2007.

39 G. Landrum, RDKIT. ORG, 2013.

40 S. Saubern, R. Guha and J. B. Baell, Mol. Inf., 2011, 30, 847850.

41 D. Lagorce, L. Bouslama, J. Becot, M. A. Miteva and B. O. Villoutreix, Bioinformatics, 2017, 33, 3658-3660.
42 R. A. Friesner, R. B. Murphy, M. P. Repasky, L. L. Frye, J. R. Greenwood, T. A. Halgren, P. C. Sanschagrin and D. T. Mainz, J. Med. Chem., 2006, 49, 6177-6196.

43 H. Wang, R. Aslanian and V. S. Madison, J. Mol. Graphics Modell., 2008, 27, 512-521.

44 R. A. Friesner, J. L. Banks, R. B. Murphy, T. A. Halgren, J. J. Klicic, D. T. Mainz, M. P. Repasky, E. H. Knoll, M. Shelley and J. K. Perry, J. Med. Chem., 2004, 47, 17391749.

45 N. Huang, C. Kalyanaraman, J. J. Irwin and M. P. Jacobson, J. Chem. Inf. Model., 2006, 46, 243-253.

46 M. Jin, N. Shepardson, T. Yang, G. Chen, D. Walsh and D. J. Selkoe, Proc. Natl. Acad. Sci. U. S. A., 2011, 108, 58195824.

47 Z. Yu, M. P. Jacobson and R. A. Friesner, J. Comput. Chem., 2006, 27, 72-89.

48 P. D. Lyne, M. L. Lamb and J. C. Saeh, J. Med. Chem., 2006, 49, 4805-4808.

49 G. M. Morris, D. S. Goodsell, R. S. Halliday, R. Huey, W. E. Hart, R. K. Belew and A. J. Olson, J. Comput. Chem., 1998, 19, 1639-1662.

50 E. M. Duffy and W. L. Jorgensen, J. Am. Chem. Soc., 2000, 122, 2878-2888.

51 C. A. Lipinski, F. Lombardo, B. W. Dominy and P. J. Feeney, Adv. Drug Delivery Rev., 1997, 23, 3-25.

52 F. Ntie-Kang, SpringerPlus, 2013, 2, 353.

53 J. Matysiak, Eur. J. Med. Chem., 2007, 42, 940-947.

54 P. M. Gill, B. G. Johnson, J. A. Pople and M. J. Frisch, Chem. Phys. Lett., 1992, 197, 499-505.

55 P. Stephens, F. Devlin, C. Chabalowski and M. J. Frisch, J. Phys. Chem., 1994, 98, 11623-11627.

56 P. Masson, W. Xie, M.-T. Froment, V. Levitsky, P.-L. Fortier, C. Albaret and O. Lockridge, Protein Struct. Mol. Enzymol., 1999, 1433, 281-293.

57 H. Motulsky, GraphPad Software, 2007, vol. 31, pp. 39-42.

58 Y. Wang, X.-L. Guan, P.-F. Wu, C.-M. Wang, H. Cao, L. Li, X.-J. Guo, F. Wang, N. Xie and F.-C. Jiang, J. Med. Chem., 2012, 55, 3588-3592.

59 R. A. Copeland, Evaluation of enzyme inhibitors in drug discovery: a guide for medicinal chemists and pharmacologists, John Wiley \& Sons, 2013.

60 J. Eichler, A. Anselment, J. L. Sussman, J. Massoulié and I. Silman, Mol. Pharmacol., 1994, 45, 335-340.

61 D. Kumar, S. K. Gupta, A. Ganeshpurkar, G. Gutti, S. Krishnamurthy, G. Modi and S. K. Singh, Eur. J. Med. Chem., 2018, 150, 87-101.

62 J. van Meerloo, G. J. L. Kaspers and J. Cloos, in Cancer Cell Culture: Methods and Protocols, ed. I. A.Cree, Humana Press, Totowa, NJ, 2011, pp. 237-245, DOI: 10.1007/978-161779-080-5_20.

63 X. Di, J. Yan, Y. Zhao, J. Zhang, Z. Shi, Y. Chang and B. Zhao, Neuroscience, 2010, 168, 778-786.

64 W. L. Jorgensen, D. S. Maxwell and J. Tirado-Rives, J. Am. Chem. Soc., 1996, 118, 11225-11236.

65 G. A. Kaminski, R. A. Friesner, J. Tirado-Rives and W. L. Jorgensen, J. Phys. Chem. B, 2001, 105, 6474-6487. 
66 U. Essmann, L. Perera, M. L. Berkowitz, T. Darden, H. Lee and L. G. Pedersen, J. Chem. Phys., 1995, 103, 8577-8593.

67 W. G. Hoover, Phys. Rev. A: At., Mol., Opt. Phys., 1985, 31, 1695.
68 G. J. Martyna, D. J. Tobias and M. L. Klein, J. Chem. Phys., 1994, 101, 4177-4189.

69 S. J. Basha, P. Mohan, D. P. Yeggoni, Z. R. Babu, P. B. Kumar, A. D. Rao, R. Subramanyam and A. G. Damu, Mol. Pharm., 2018, 15, 2206-2223. 